\title{
Lagrangian mapping class groups from a group homological point of view
}

\author{
TAKUYA SAKASAI
}

\begin{abstract}
We focus on two kinds of infinite index subgroups of the mapping class group of a surface associated with a Lagrangian submodule of the first homology of a surface. These subgroups, called Lagrangian mapping class groups, are known to play important roles in the interaction between the mapping class group and finite-type invariants of 3-manifolds. In this paper, we discuss these groups from a group (co)homological point of view. The results include the determination of their abelianizations, lower bounds of the second homology and remarks on the (co)homology of higher degrees. As a byproduct of this investigation, we determine the second homology of the mapping class group of a surface of genus 3 .
\end{abstract}

55R40; 32G15, 57R20

\section{Introduction}

Let $\Sigma_{g}$ be a closed oriented connected surface of genus $g$ and let $H_{g}$ be an oriented handlebody of the same genus. As shown in Figure 1, put $H_{g}$ in the standard position in $\mathbb{R}^{3}$ and consider $\Sigma_{g}$ to be the boundary of $H_{g}$. Fix a basis $\left\{x_{1}, x_{2}, \ldots, x_{g}, y_{1}, y_{2}, \ldots, y_{g}\right\}$ of $H:=H_{1}\left(\Sigma_{g}\right)$ as in the figure so that $\operatorname{Ker}\left(H_{1}\left(\Sigma_{g}\right) \rightarrow H_{1}\left(H_{g}\right)\right)$ coincides with the submodule $L$ of $H$ generated by $\left\{x_{1}, x_{2}, \ldots, x_{g}\right\}$.

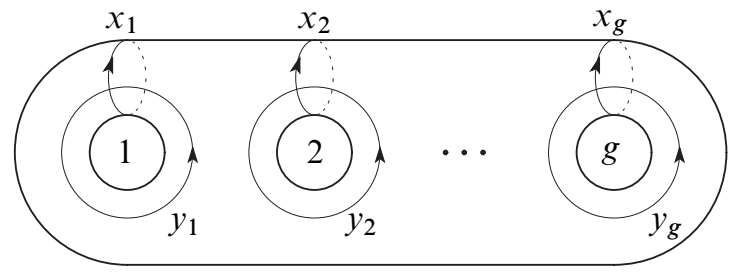

Figure 1. A symplectic basis of $H_{1}\left(\Sigma_{g}\right)$

The module $H$ has a natural nondegenerate antisymmetric bilinear form $\mu: H \otimes H \rightarrow \mathbb{Z}$ called the intersection pairing. It is easy to see that $L$ is a maximal direct summand of $H$ on which $\mu$ restricts to 0 . Such a submodule is said to be Lagrangian. By using 
the pairing $\mu$, we can naturally identify the quotient module $H / L$, the dual module $L^{*}:=\operatorname{Hom}(L, \mathbb{Z})$ and the submodule $L_{y}$ of $H$ generated by $\left\{y_{1}, y_{2}, \ldots, y_{g}\right\}$.

The mapping class group $\mathcal{M}_{g}$ of $\Sigma_{g}$ is the group of isotopy classes of orientation preserving self-diffeomorphisms of $\Sigma_{g}$. In this paper, we focus on subgroups of $\mathcal{M}_{g}$ associated with the above fixed Lagrangian submodule $L$ of $H$. More precisely, two subgroups

$$
\begin{aligned}
\mathcal{L}_{g} & :=\left\{f \in \mathcal{M}_{g} \mid f_{*}(L)=L\right\}, \\
\mathcal{I} \mathcal{L}_{g} & :=\left\{f \in \mathcal{M}_{g}\left|f_{*}\right|_{L}=\mathrm{id}_{L}\right\}
\end{aligned}
$$

are studied through their group (co)homology, where $f_{*}$ denotes the induced automorphism of $H$ for $f \in \mathcal{M}_{g}$. We have $\mathcal{I} \mathcal{L}_{g} \subset \mathcal{L}_{g}$ by definition and call them Lagrangian mapping class groups or Lagrangian subgroups. The Torelli group $\mathcal{I}_{g}$ is defined by

$$
\mathcal{I}_{g}:=\left\{f \in \mathcal{M}_{g} \mid f_{*}=\operatorname{id}_{H}\right\} .
$$

One motivation by which the author started to study the groups $\mathcal{L}_{g}$ and $\mathcal{I} \mathcal{L}_{g}$ is the fact that they are infinite index subgroups of $\mathcal{M}_{g}$ including $\mathcal{I}_{g}$. The importance to study this kind of subgroups will be explained in Section 7.2 with the relationship to the (non)triviality problem of even Miller-Morita-Mumford classes $e_{2 i} \in H^{4 i}\left(\mathcal{M}_{g} ; \mathbb{Q}\right)$ pulled back to $H^{4 i}\left(\mathcal{I}_{g} ; \mathbb{Q}\right)$.

Lagrangian subgroups have been studied by several researchers. Hirose studied a generating system of $\mathcal{L}_{g}$ in [16], where $\mathcal{L}_{g}$ is called the homological handlebody group. In fact, the group $\mathcal{L}_{g}$ can be seen as a homological extension of the handlebody mapping class group $\mathcal{H}_{g}$. Recall that the group $\mathcal{H}_{g}$ is the subgroup of $\mathcal{M}_{g}$ consisting of isotopy classes of orientation preserving self-diffeomorphisms of $\Sigma_{g}=\partial H_{g}$ that can be extended to self-diffeomorphisms of the handlebody $H_{g}$. We can easily check that $\mathcal{L}_{g}=\mathcal{H}_{g} \mathcal{I}_{g}$. Prior to Hirose's work, Birman gave a generating set of $\mathcal{L}_{g} / \mathcal{I}_{g} \cong$ $\mathcal{H}_{g} /\left(\mathcal{H}_{g} \cap \mathcal{I}_{g}\right)$ in [4] and we can give a generating set of $\mathcal{L}_{g}$ by combining her result with Johnson's finite generating set of $\mathcal{I}_{g}$ [19].

As for $\mathcal{I} \mathcal{L}_{g}$, Levine conducted a series of investigations in [23; 24; 25]. He defined a filtration of $\mathcal{I} \mathcal{L}_{g}$ called the Lagrangian filtration, which is analogous to the Johnson filtration of $\mathcal{I}_{g}$, by modifying the theory of Johnson homomorphisms so that it conforms well to $\mathcal{I} \mathcal{L}_{g}$. Then he gave an application of this filtration to the theory of homology 3-spheres.

Recently, the groups $\mathcal{L}_{g}$ and $\mathcal{I} \mathcal{L}_{g}$ appear and play important roles in the theory of finite-type invariants of 3-manifolds. See Andersen, Bene, Meilhan and Penner [1], Cheptea, Habiro and Massuyeau [10], Cheptea and Le [11] (with a slightly different definition) and Garoufalidis and Levine [13], for example. However, it seems that the 
groups $\mathcal{L}_{g}$ and $\mathcal{I} \mathcal{L}_{g}$ have been studied separately. In this paper, we put $\mathcal{L}_{g}$ on the top of the Lagrangian filtration of $\mathcal{I} \mathcal{L}_{g}$ and study them simultaneously as in the case of $\mathcal{M}_{g}$ and $\mathcal{I}_{g}$.

We first summarize the notation and fundamental facts on $\mathcal{L}_{g}$ and $\mathcal{I} \mathcal{L}_{g}$ in Section 2. Then we will discuss the following in order.

- Section 3: Computation of $H_{1}\left(\mathcal{I} \mathcal{L}_{g}\right)$

- Section 4: Computations of $H_{1}\left(\mathcal{L}_{g} / \mathcal{I}_{g}\right)$ and $H_{2}\left(\mathcal{L}_{g} / \mathcal{I}_{g}\right)$

- Section 5: Computation of $H_{1}\left(\mathcal{L}_{g}\right)$ and a lower bound of $H_{2}\left(\mathcal{L}_{g}\right)$

- Section 7: Remarks on higher (co)homology of $\mathcal{L}_{g}$ and $\mathcal{I} \mathcal{L}_{g}$

Precisely speaking, we study in Sections 3 and 5 the Lagrangian mapping class groups of a surface with one boundary component and then derive the statements for those of a closed surface in Section 6.

As a byproduct, we will give a remark that the second homology of the full mapping class group of genus 3 has $\mathbb{Z}_{2}$ as a direct summand (Theorem 4.9 and Corollary 4.10). This homology group has been almost determined by Korkmaz and Stipsicz [22] up to this $\mathbb{Z}_{2}$ summand.

In this paper, we use the same notation $H_{*}(\cdot)$ for the homology of both topological spaces and groups unless otherwise stated. We refer to Brown's book [9] for generalities of group (co)homology.

\section{Lagrangian mapping class groups}

By using the ordered basis $\left\{x_{1}, x_{2}, \ldots, x_{g}, y_{1}, y_{2}, \ldots, y_{g}\right\}$ of $H$, we fix an isomorphism between $\mathbb{Z}^{2 g}$ and $H$, which enables us to identify the symplectic group $\operatorname{Sp}(2 g, \mathbb{Z})$ with the group of automorphisms of $H$ preserving the intersection pairing $\mu$. Then the action of $\mathcal{M}_{g}$ on $H$ gives the exact sequence

$$
1 \longrightarrow \mathcal{I}_{g} \longrightarrow \mathcal{M}_{g} \stackrel{\sigma}{\longrightarrow} \operatorname{Sp}(2 g, \mathbb{Z}) \longrightarrow 1
$$

with $\operatorname{Ker} \sigma=\mathcal{I}_{g}$, the Torelli group. The symplecticity condition for a $(2 g) \times(2 g)$ matrix

$$
X=\left(\begin{array}{ll}
A & B \\
C & D
\end{array}\right)
$$

with $g \times g$ matrices $A, B, C, D$ is given by

$$
{ }^{t} X\left(\begin{array}{cc}
O & I_{g} \\
-I_{g} & O
\end{array}\right) X=\left(\begin{array}{cc}
O & I_{g} \\
-I_{g} & O
\end{array}\right),
$$


where we denote by $I_{g}$ the identity matrix of size $g$. The left hand side is equal to

$$
\left(\begin{array}{l}
-{ }^{t} C A+{ }^{t} A C-{ }^{t} C B+{ }^{t} A D \\
-{ }^{t} D A+{ }^{t} B C-{ }^{t} D B+{ }^{t} B D
\end{array}\right) .
$$

From this we see that if $C=O$, then $D={ }^{t} A^{-1}$ holds and $A^{-1} B$ is symmetric. This case corresponds to $\sigma\left(\mathcal{L}_{g}\right)$. That is, if we put

$$
\operatorname{urSp}(2 g):=\left\{\left(\begin{array}{cc}
A & B \\
O & { }^{t} A^{-1}
\end{array}\right) \mid A^{-1} B: \text { symmetric }\right\},
$$

then it is a subgroup of $\operatorname{Sp}(2 g, \mathbb{Z})$ and the equality $\mathcal{L}_{g}=\sigma^{-1}(\operatorname{urSp}(2 g))$ follows by definition. The notation urSp $(2 g)$ meaning "upper right" was introduced by Hirose [16]. We have the exact sequence

$$
1 \longrightarrow \mathcal{I}_{g} \longrightarrow \mathcal{L}_{g} \stackrel{\left.\sigma\right|_{\mathcal{L} g}}{\longrightarrow} \operatorname{urSp}(2 g) \longrightarrow 1 .
$$

Moreover, if $C=O$ and $A=D=I_{g}$, then the matrix $B$ itself is symmetric. In this case, the subgroup

$$
\left\{\left(\begin{array}{cc}
I_{g} & B \\
O & I_{g}
\end{array}\right) \mid B: \text { symmetric }\right\}
$$

is naturally isomorphic to the second symmetric power $S^{2} L$ of $L$ because

$$
\operatorname{Hom}\left(L_{y}, L\right) \cong \operatorname{Hom}\left(L^{*}, L\right) \cong L \otimes L
$$

and $B$ is symmetric. By definition, the equality $\mathcal{I} \mathcal{L}_{g}=\sigma^{-1}\left(S^{2} L\right)$ holds and we have the exact sequence

$$
1 \longrightarrow \mathcal{I}_{g} \longrightarrow \mathcal{I L}_{g} \stackrel{\left.\sigma\right|_{\mathcal{I} \mathcal{L}_{g}}}{\longrightarrow} S^{2} L \longrightarrow 1 \text {. }
$$

Note that $S^{2} L$ is a free abelian group. The groups $S^{2} L$ and $\operatorname{urSp}(2 g)$ are related by the exact sequence

$$
1 \longrightarrow S^{2} L \longrightarrow \operatorname{urSp}(2 g) \stackrel{\mathrm{ul}}{\longrightarrow} \mathrm{GL}(g, \mathbb{Z}) \longrightarrow 1,
$$

where the map ul assigns to each matrix its upper left block of size $g \times g$. Note that this group extension has a splitting defined by

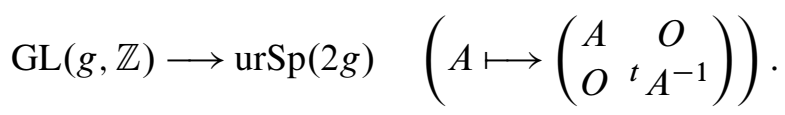

Using (4), we obtain the exact sequence

$$
1 \longrightarrow \mathcal{I} \mathcal{L}_{g} \longrightarrow \mathcal{L}_{g} \stackrel{\left.\mathrm{ul} \circ \sigma\right|_{\mathcal{L}_{g}}}{\longrightarrow} \operatorname{GL}(g, \mathbb{Z}) \longrightarrow 1 .
$$


In the subsequent sections, we will use the above exact sequences to discuss the homology of $\mathcal{L}_{g}$ and $\mathcal{I} \mathcal{L}_{g}$. By a technical reason, however, we first consider the mapping class group $\mathcal{M}_{g, 1}$ of the surface $\Sigma_{g, 1}$ obtained from $\Sigma_{g}$ by removing an open disk, where each mapping class is supposed to fix the boundary of $\Sigma_{g, 1}$ pointwise. The subgroups $\mathcal{L}_{g, 1}, \mathcal{I} \mathcal{L}_{g, 1}$ and $\mathcal{I}_{g, 1}$ are defined similarly. Exact sequences similar to the above hold for these groups. We naturally identify $H$ with $H_{1}\left(\Sigma_{g, 1}\right)$. Also, we assume that $g \geq 3$ to avoid the complexity of $\mathcal{I}_{2,1}$, which is not covered by Johnson's work (see the next section).

\section{The first homology of $\mathcal{I} \mathcal{L}_{g, 1}$}

We begin our investigation by determining the first homology, namely the abelianization, of $\mathcal{I} \mathcal{L}_{g, 1}$. For that, we use the five-term exact sequence

$$
H_{2}\left(\mathcal{I} \mathcal{L}_{g, 1}\right) \rightarrow H_{2}\left(S^{2} L\right) \rightarrow H_{1}\left(\mathcal{I}_{g, 1}\right)_{S^{2} L} \rightarrow H_{1}\left(\mathcal{I} \mathcal{L}_{g, 1}\right) \rightarrow H_{1}\left(S^{2} L\right) \rightarrow 0
$$

associated with the group extension (3). Put

$$
X_{i}^{2}:=x_{i} \otimes x_{i}, \quad X_{i j}=X_{j i}:=x_{i} \otimes x_{j}+x_{j} \otimes x_{i} .
$$

The set

$$
\left\{X_{i}^{2} \mid 1 \leq i \leq g\right\} \cup\left\{X_{i j} \mid 1 \leq i<j \leq g\right\}
$$

forms a basis of $S^{2} L$ in $L \otimes L$. As a subgroup of $\operatorname{Sp}(2 g, \mathbb{Z})$, the group $S^{2} L$ acts on $H$ by

$$
X_{i}^{2}:\left\{\begin{array}{l}
x_{k} \mapsto x_{k}, \\
y_{k} \mapsto \delta_{i k} x_{i}+y_{k},
\end{array} \quad X_{i j}:\left\{\begin{array}{l}
x_{k} \mapsto x_{k}, \\
y_{k} \mapsto \delta_{j k} x_{i}+\delta_{i k} x_{j}+y_{k},
\end{array}\right.\right.
$$

where $\delta_{i j}$ is the Kronecker delta.

Lemma 3.1 The homomorphism $\left(\left.\sigma\right|_{\mathcal{I} \mathcal{L}_{g, 1}}\right)_{*}: H_{2}\left(\mathcal{I} \mathcal{L}_{g, 1}\right) \rightarrow H_{2}\left(S^{2} L\right) \cong \wedge^{2}\left(S^{2} L\right)$ is surjective.

Proof We use the technique of abelian cycles to construct homology classes in $\operatorname{Im}\left(\left.\sigma\right|_{\mathcal{I} \mathcal{L}_{g, 1}}\right)_{*}$. That is, for each homomorphism $\varphi: \mathbb{Z}^{2} \rightarrow \mathcal{I} \mathcal{L}_{g, 1}$, we have a homology class $\varphi_{*}(1) \in H_{2}\left(\mathcal{I} \mathcal{L}_{g, 1}\right)$ by sending the fundamental class $1 \in H_{2}\left(\mathbb{Z}^{2}\right) \cong \mathbb{Z}$ to $H_{2}\left(\mathcal{I} \mathcal{L}_{g, 1}\right)$. Such a class $\varphi_{*}(1)$, which is in fact defined on cycle level, is called an abelian cycle associated with $\varphi$. Moreover, we can see that

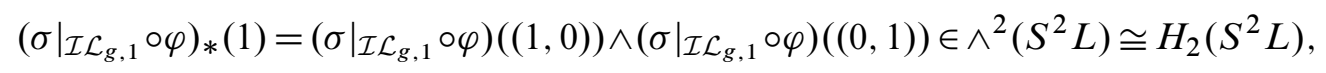

where $(1,0),(0,1) \in \mathbb{Z}^{2}$ (see [32, Lemma 2.2] for details). 

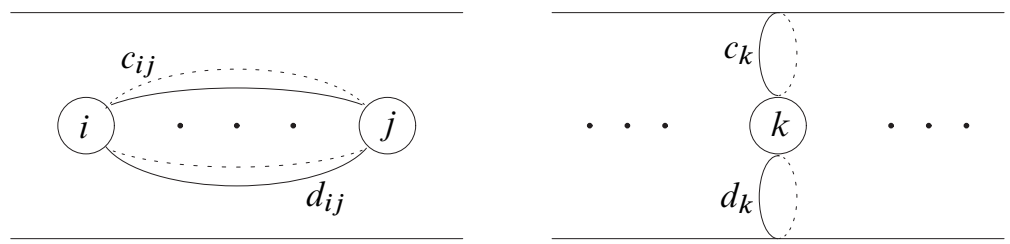

Figure 2

Define simple closed curves $c_{i j}, d_{i j}, c_{k}, d_{k}(1 \leq i<j \leq g, 1 \leq k \leq g)$ on $\Sigma_{g, 1}$ as in Figure 2. Let $G_{1}$ (resp. $G_{2}$ ) be the subgroup of $\mathcal{I} \mathcal{L}_{g}$ generated by $\left\{T_{c_{i j}}\right\}_{i, j} \cup\left\{T_{c_{k}}\right\}_{k}$ (resp. $\left\{T_{d_{i j}}\right\}_{i, j} \cup\left\{T_{d_{k}}\right\}_{k}$ ), where $T_{c}$ denotes the right-handed Dehn twist along a simple closed curve $c$. Since

$\left.\sigma\right|_{\mathcal{I} \mathcal{L}_{g, 1}}\left(T_{c_{i j}}\right)=\left.\sigma\right|_{\mathcal{I} \mathcal{L}_{g, 1}}\left(T_{d_{i j}}\right)=X_{i}^{2}-X_{i j}+X_{j}^{2},\left.\quad \sigma\right|_{\mathcal{I} \mathcal{L}_{g, 1}}\left(T_{c_{k}}\right)=\left.\sigma\right|_{\mathcal{I} \mathcal{L}_{g, 1}}\left(T_{d_{k}}\right)=X_{k}^{2}$,

each of $\left.\sigma\right|_{\mathcal{I} \mathcal{L}_{g, 1}}\left(G_{1}\right)$ and $\left.\sigma\right|_{\mathcal{I} \mathcal{L}_{g, 1}}\left(G_{2}\right)$ generates $S^{2} L$. Clearly $f g=g f \in \mathcal{I} \mathcal{L}_{g}$ holds for any $f \in G_{1}$ and $g \in G_{2}$. Hence, for each element of the form $a \wedge b$ in $\wedge^{2}\left(S^{2} L\right)$, we can take $f_{1} \in G_{1}$ and $f_{2} \in G_{2}$ satisfying

$$
\left(\left.\sigma\right|_{\mathcal{I} \mathcal{L}_{g, 1}}\right)\left(f_{1}\right)=a, \quad\left(\left.\sigma\right|_{\mathcal{I} \mathcal{L}_{g, 1}}\right)\left(f_{2}\right)=b, \quad f_{1} f_{2}=f_{2} f_{1} .
$$

They give a homomorphism $\varphi: \mathbb{Z}^{2} \rightarrow \mathcal{I} \mathcal{L}_{g, 1}$ with $\left(\left.\sigma\right|_{\mathcal{I} \mathcal{L}_{g, 1}} \circ \varphi\right)_{*}(1)=a \wedge b$, which implies the surjectivity of $\left(\left.\sigma\right|_{\mathcal{I} \mathcal{L}_{g, 1}}\right)_{*}$.

Lemma 3.1 shows that $H_{2}\left(\mathcal{I} \mathcal{L}_{g, 1}\right)$ is nontrivial (see also Theorem 7.1). In particular, its rank, which may be infinite, gets bigger and bigger when $g$ grows.

Before going further, here we recall some results on the Torelli group $\mathcal{I}_{g, 1}$ obtained by Johnson in $[17 ; 18 ; 19 ; 20 ; 21]$. First, he showed in [19] that $\mathcal{I}_{g, 1}$ is finitely generated for $g \geq 3$. This fact together with the sequences (2), (3) imply that $\mathcal{L}_{g, 1}$ and $\mathcal{I} \mathcal{L}_{g, 1}$ are also finitely generated. At present, it is not known whether they are finitely presentable or not, where the same question for $\mathcal{I}_{g, 1}$ is a well-known open problem. Second, he showed that $\mathcal{I}_{g, 1}$ is normally generated by only one element $T_{c_{2}} T_{d_{2}}^{-1}$ (see Figure 2). Finally, in [21], he determined the abelianization of $\mathcal{I}_{g, 1}$ written as follows. Let $B$ be a commutative $\mathbb{Z}_{2}$-algebra with unit 1 generated by formal elements $\bar{x}$ for $x \in H \otimes \mathbb{Z}_{2}$ and having relations

$$
\bar{x}^{2}=\bar{x}, \quad \overline{x+y}=\bar{x}+\bar{y}+\bar{\mu}(x, y)
$$

for $x, y \in H \otimes \mathbb{Z}_{2}$, where $\bar{\mu}(x, y):=\mu(x, y) \bmod 2$. The algebra $B$ can be graded by supposing that each $\bar{x}$ has degree 1 (after replacing $\bar{x}^{2}$ by $\bar{x}$ ). Let $B^{i}$ be the 
submodule of $B$ generated by elements of degree at most $i$. This endows $B$ with a filtration

$$
B^{3} \supset B^{2} \supset B^{1} \supset B^{0}=\{0,1\} .
$$

We have a natural action of $\mathcal{M}_{g, 1}$ on $B^{3}$ defined by $f \bar{x}:=\overline{f_{*}(x)}$. It is easily checked that there exists a natural $\mathcal{M}_{g, 1}$-equivariant isomorphism

$$
B^{3} / B^{2} \cong \wedge^{3}\left(H \otimes \mathbb{Z}_{2}\right) .
$$

Therefore we can take the fiber product $\wedge^{3} H \times \wedge^{3}\left(H \otimes \mathbb{Z}_{2}\right) B^{3}$ of the natural projections $B^{3} \rightarrow B^{3} / B^{2} \cong \wedge^{3}\left(H \otimes \mathbb{Z}_{2}\right)$ and $\wedge^{3} H \rightarrow \wedge^{3}\left(H \otimes \mathbb{Z}_{2}\right)$. Then Johnson gave an $\mathcal{M}_{g, 1}$-equivariant isomorphism

$$
(\tau, \beta): H_{1}\left(\mathcal{I}_{g, 1}\right) \stackrel{\cong}{\longrightarrow} \wedge^{3} H \times \wedge^{3}\left(H \otimes \mathbb{Z}_{2}\right) B^{3},
$$

where $\mathcal{M}_{g, 1}$ acts on $\mathcal{I}_{g, 1}$ and $H_{1}\left(\mathcal{I}_{g, 1}\right)$ by conjugation and on $\wedge^{3} H \times \wedge^{3}\left(H \otimes \mathbb{Z}_{2}\right) B^{3}$ diagonally. The homomorphism $\tau$ is now called the Johnson homomorphism [17; 20] and $\beta$ is called the Birman-Craggs-Johnson homomorphism (see Johnson [18] and Birman and Craggs [6]). Explicitly, the isomorphism is given by

$$
T_{c_{2}} T_{d_{2}}^{-1} \longmapsto\left(x_{1} \wedge y_{1} \wedge y_{2}, \bar{x}_{1} \bar{y}_{1}\left(\bar{y}_{2}+1\right)\right),
$$

which characterizes an $\mathcal{M}_{g, 1}$-equivariant homomorphism uniquely because $\mathcal{I}_{g, 1}$ is normally generated by $T_{c_{2}} T_{d_{2}}^{-1}$.

Lemma 3.2 We have the following isomorphism:

$$
H_{1}\left(\mathcal{I}_{g, 1}\right)_{S^{2} L} \cong \begin{cases}\wedge^{3} L^{*} \oplus L^{*} \oplus \wedge^{2}\left(L^{*} \otimes \mathbb{Z}_{2}\right) & g=3, \\ \wedge^{3} L^{*} \oplus L^{*} & g \geq 4\end{cases}
$$

Proof By definition, the coinvariant part $H_{1}\left(\mathcal{I}_{g, 1}\right)_{S^{2} L}$ is the quotient of $H_{1}\left(\mathcal{I}_{g, 1}\right)$ by the submodule $Q_{0}$ generated by $\left\{\sigma x-x \mid \sigma \in S^{2} L, x \in H_{1}\left(\mathcal{I}_{g, 1}\right)\right\}$. We now list a generating set of $Q_{0}$ explicitly. Assuming that the indices $i, j, k, l \in\{1,2, \ldots, g\}$ are distinct from each other, we have

$$
\begin{aligned}
X_{j}^{2}\left(x_{i} \wedge x_{j} \wedge y_{j}, \bar{x}_{i}\right. & \left.\bar{x}_{j} \bar{y}_{j}\right)-\left(x_{i} \wedge x_{j} \wedge y_{j}, \bar{x}_{i} \bar{x}_{j} \bar{y}_{j}\right) \\
& =\left(x_{i} \wedge x_{j} \wedge\left(x_{j}+y_{j}\right), \bar{x}_{i} \bar{x}_{j} \bar{x}_{j}+y_{j}\right)-\left(x_{i} \wedge x_{j} \wedge y_{j}, \bar{x}_{i} \bar{x}_{j} \bar{y}_{j}\right) \\
& =\left(x_{i} \wedge x_{j} \wedge y_{j}, \bar{x}_{i} \bar{x}_{j}\left(\bar{x}_{j}+\bar{y}_{j}+1\right)\right)-\left(x_{i} \wedge x_{j} \wedge y_{j}, \bar{x}_{i} \bar{x}_{j} \bar{y}_{j}\right) \\
& =\left(0, \bar{x}_{i} \bar{x}_{j}^{2}+\bar{x}_{i} \bar{x}_{j}\right)=(0,0),
\end{aligned}
$$

where we used the relations $\bar{x}_{j}^{2}=\bar{x}_{j}$ and $2 \bar{x}_{i} \bar{x}_{j}=0$ in $B^{3}$. We denote this result by

$$
\left[X_{j}^{2} ;\left(x_{i} \wedge x_{j} \wedge y_{j}, \bar{x}_{i} \bar{x}_{j} \bar{y}_{j}\right)\right]:=(0,0)
$$


for short. Similar calculations show that

(1b) $\left[X_{k j} ;\left(x_{i} \wedge x_{j} \wedge y_{j}, \bar{x}_{i} \bar{x}_{j} \bar{y}_{j}\right)\right]=\left(x_{i} \wedge x_{j} \wedge x_{k}, \bar{x}_{i} \bar{x}_{j} \bar{x}_{k}\right)$,

(1c) $\left[X_{i j} ;\left(x_{i} \wedge x_{j} \wedge y_{j}, \bar{x}_{i} \bar{x}_{j} \bar{y}_{j}\right)\right]=\left(0, \bar{x}_{i} \bar{x}_{j}\right)$,

(2a) $\left[X_{k}^{2} ;\left(x_{i} \wedge x_{j} \wedge y_{k}, \bar{x}_{i} \bar{x}_{j} \bar{y}_{k}\right)\right]=\left(x_{i} \wedge x_{j} \wedge x_{k}, \bar{x}_{i} \bar{x}_{j} \bar{x}_{k}+\bar{x}_{i} \bar{x}_{j}\right)$,

(2b) $\left[X_{j k} ;\left(x_{i} \wedge x_{j} \wedge y_{k}, \bar{x}_{i} \bar{x}_{j} \bar{y}_{k}\right)\right]=\left(0, \bar{x}_{i} \bar{x}_{j}\right)$,

$(2 \mathrm{c})^{*}\left[X_{l k} ;\left(x_{i} \wedge x_{j} \wedge y_{k}, \bar{x}_{i} \bar{x}_{j} \bar{y}_{k}\right)\right]=\left(x_{i} \wedge x_{j} \wedge x_{l}, \bar{x}_{i} \bar{x}_{j} \bar{x}_{l}\right)$,

(3a) $\left[X_{j}^{2} ;\left(x_{i} \wedge y_{i} \wedge y_{j}, \bar{x}_{i} \bar{y}_{i} \bar{y}_{j}\right)\right]=\left(-x_{i} \wedge x_{j} \wedge y_{i}, \bar{x}_{i} \bar{x}_{j} \bar{y}_{i}+\bar{x}_{i} \bar{y}_{i}\right)$,

(3b) $\left[X_{i k} ;\left(x_{i} \wedge y_{i} \wedge y_{j}, \bar{x}_{i} \bar{y}_{i} \bar{y}_{j}\right)\right]=\left(x_{i} \wedge x_{k} \wedge y_{j}, \bar{x}_{i} \bar{x}_{k} \bar{y}_{j}\right)$,

(3c) $\left[X_{j k} ;\left(x_{i} \wedge y_{i} \wedge y_{j}, \bar{x}_{i} \bar{y}_{i} \bar{y}_{j}\right)\right]=\left(-x_{i} \wedge x_{k} \wedge y_{i}, \bar{x}_{i} \bar{x}_{k} \bar{y}_{i}\right)$,

(3d) $\left[X_{i j} ;\left(x_{i} \wedge y_{i} \wedge y_{j}, \bar{x}_{i} \bar{y}_{i} \bar{y}_{j}\right)\right]=\left(x_{i} \wedge x_{j} \wedge y_{j}, \bar{x}_{i} \bar{x}_{j} \bar{y}_{j}+\bar{x}_{i} \bar{x}_{j}+\bar{x}_{i} \bar{y}_{i}\right)$,

(4a) $\left[X_{j}^{2} ;\left(x_{i} \wedge y_{j} \wedge y_{k}, \bar{x}_{i} \bar{y}_{j} \bar{y}_{k}\right)\right]=\left(x_{i} \wedge x_{j} \wedge y_{k}, \bar{x}_{i} \bar{x}_{j} \bar{y}_{k}+\bar{x}_{i} \bar{y}_{k}\right)$,

(4b) $\left[X_{i j} ;\left(x_{i} \wedge y_{j} \wedge y_{k}, \bar{x}_{i} \bar{y}_{j} \bar{y}_{k}\right)\right]=\left(0, \bar{x}_{i} \bar{y}_{k}\right)$,

$(4 \mathrm{c})^{*}\left[X_{j l} ;\left(x_{i} \wedge y_{j} \wedge y_{k}, \bar{x}_{i} \bar{y}_{j} \bar{y}_{k}\right)\right]=\left(x_{i} \wedge x_{l} \wedge y_{k}, \bar{x}_{i} \bar{x}_{l} \bar{y}_{k}\right)$,

(4d) $\left[X_{j k} ;\left(x_{i} \wedge y_{j} \wedge y_{k}, \bar{x}_{i} \bar{y}_{j} \bar{y}_{k}\right)\right]=\left(x_{i} \wedge x_{k} \wedge x_{j}+x_{i} \wedge x_{k} \wedge y_{k}-x_{i} \wedge x_{j} \wedge y_{j}\right.$, $\left.\bar{x}_{i} \bar{x}_{k} \bar{x}_{j}+\bar{x}_{i} \bar{x}_{k} \bar{y}_{k}+\bar{x}_{i} \bar{x}_{j} \bar{y}_{j}\right)$

(5a) $\left[X_{i}^{2} ;\left(y_{i} \wedge y_{j} \wedge y_{k}, \bar{y}_{i} \bar{y}_{j} \bar{y}_{k}\right)\right]=\left(x_{i} \wedge y_{j} \wedge y_{k}, \bar{x}_{i} \bar{y}_{j} \bar{y}_{k}+\bar{y}_{j} \bar{y}_{k}\right)$,

$(5 \mathrm{~b})^{*}\left[X_{i l} ;\left(y_{i} \wedge y_{j} \wedge y_{k}, \bar{y}_{i} \bar{y}_{j} \bar{y}_{k}\right)\right]=\left(x_{l} \wedge y_{j} \wedge y_{k}, \bar{x}_{l} \bar{y}_{j} \bar{y}_{k}\right)$,

(5c) $\left[X_{i j} ;\left(y_{i} \wedge y_{j} \wedge y_{k}, \bar{y}_{i} \bar{y}_{j} \bar{y}_{k}\right)\right]=\left(x_{j} \wedge x_{i} \wedge y_{k}+x_{j} \wedge y_{j} \wedge y_{k}-x_{i} \wedge y_{i} \wedge y_{k}\right.$, $\left.\bar{x}_{j} \bar{x}_{i} \bar{y}_{k}+\bar{x}_{j} \bar{y}_{j} \bar{y}_{k}+\bar{x}_{i} \bar{y}_{i} \bar{y}_{k}\right)$,

(6) $\left[X_{i j} ;\left(0, \bar{x}_{i} \bar{y}_{i}\right)\right]=\left(0, \bar{x}_{i} \bar{x}_{j}\right)$,

(7a) $\left[X_{j}^{2} ;\left(0, \bar{x}_{i} \bar{y}_{j}\right)\right]=\left(0, \bar{x}_{i} \bar{x}_{j}+\bar{x}_{i}\right)$,

(7b) $\left[X_{i j} ;\left(0, \bar{x}_{i} \bar{y}_{j}\right)\right]=\left(0, \bar{x}_{i}\right)$,

(7c) $\left[X_{j k} ;\left(0, \bar{x}_{i} \bar{y}_{j}\right)\right]=\left(0, \bar{x}_{i} \bar{x}_{k}\right)$,

(8a) $\left[X_{i}^{2} ;\left(0, \bar{y}_{i} \bar{y}_{j}\right)\right]=\left(0, \bar{x}_{i} \bar{y}_{j}+\bar{y}_{j}\right)$,

(8b) $\left[X_{i k} ;\left(0, \bar{y}_{i} \bar{y}_{j}\right)\right]=\left(0, \bar{x}_{k} \bar{y}_{j}\right)$,

(8c) $\left[X_{i j} ;\left(0, \bar{y}_{i} \bar{y}_{j}\right)\right]=\left(0, \bar{x}_{i} \bar{x}_{j}+\bar{x}_{i} \bar{y}_{i}+\bar{x}_{j} \bar{y}_{j}\right)$,

(9a) $\left[X_{i}^{2} ;\left(0, \bar{y}_{i}\right)\right]=\left(0, \bar{x}_{i}+1\right)$,

(9b) $\left[X_{i j} ;\left(0, \bar{y}_{i}\right)\right]=\left(0, \bar{x}_{j}\right)$, 
where $(\cdot)^{*}$ means that it is valid for $g \geq 4$. The actions not listed above are all trivial, namely $\sigma x-x=(0,0)$, so that they do not contribute to $Q_{0}$. In particular, there are no contribution from the elements

$$
\left(x_{i} \wedge x_{j} \wedge x_{k}, \bar{x}_{i} \bar{x}_{j} \bar{x}_{k}\right), \quad\left(0, \bar{x}_{i} \bar{x}_{j}\right), \quad\left(0, \bar{x}_{i}\right), \quad(0,1) .
$$

From (7b), (9a), (1c), (1b), (4b), (8a), (3b), (3c), (3a), (5b), (5a), we see that, for $g \geq 4$, $Q_{0}$ contains $\left(0, \bar{x}_{j}\right),(0,1),\left(0, \bar{x}_{i} \bar{x}_{j}\right),\left(x_{i} \wedge x_{j} \wedge x_{k}, \bar{x}_{i} \bar{x}_{j} \bar{x}_{k}\right),\left(0, \bar{x}_{i} \bar{y}_{k}\right),\left(0, \bar{y}_{j}\right)$, $\left(x_{i} \wedge x_{k} \wedge y_{j}, \bar{x}_{i} \bar{x}_{k} \bar{y}_{j}\right),\left(x_{i} \wedge x_{k} \wedge y_{i}, \bar{x}_{i} \bar{x}_{k} \bar{y}_{i}\right),\left(0, \bar{x}_{i} \bar{y}_{i}\right),\left(x_{l} \wedge y_{j} \wedge y_{k}, \bar{x}_{l} \bar{y}_{j} \bar{y}_{k}\right)$, $\left(0, \bar{y}_{j} \bar{y}_{k}\right)$ in order, and combinations of these elements express all the generators listed above except (5c). Finally (5c) shows $\left(x_{j} \wedge y_{j} \wedge y_{k}-x_{i} \wedge y_{i} \wedge y_{k}, \bar{x}_{j} \bar{y}_{j} \bar{y}_{k}+\bar{x}_{i} \bar{y}_{i} \bar{y}_{k}\right)$ are in $Q_{0}$. Our claim for $g \geq 4$ follows from this, where we assign $y_{k} \in L^{*}$ to $\left(y_{k} \wedge x_{i} \wedge y_{i}, \bar{y}_{k} \bar{x}_{i} \bar{y}_{i}\right) \in H_{1}\left(\mathcal{I}_{g, 1}\right)_{S^{2} L}$, which does not depend on $i$.

When $g=3$, differently from the above, we cannot remove $\left(x_{l} \wedge y_{j} \wedge y_{k}, \bar{x}_{l} \bar{y}_{j} \bar{y}_{k}\right)$ and $\left(0, \bar{y}_{j} \bar{y}_{k}\right)$ simultaneously. In this case, we use (5a) to eliminate $\left(x_{l} \wedge y_{j} \wedge y_{k}, \bar{x}_{l} \bar{y}_{j} \bar{y}_{k}\right)$ and conclude that $\left(0, \bar{y}_{j} \bar{y}_{k}\right)$ survive in $H_{1}\left(\mathcal{I}_{g, 1}\right)_{S^{2} L}$ and form $\wedge^{2}\left(L^{*} \otimes \mathbb{Z}_{2}\right)$.

By the exact sequence (6) together with Lemmas 3.1, 3.2, we conclude the following.

\section{Theorem 3.3}

$$
H_{1}\left(\mathcal{I} \mathcal{L}_{g, 1}\right) \cong \begin{cases}\wedge^{3} L^{*} \oplus L^{*} \oplus \wedge^{2}\left(L^{*} \otimes \mathbb{Z}_{2}\right) \oplus S^{2} L & g=3 \\ \wedge^{3} L^{*} \oplus L^{*} \oplus S^{2} L & g \geq 4\end{cases}
$$

Remark 3.4 In [23, Theorem 1], Levine constructed a surjective homomorphism

$$
\mathcal{J}: H_{1}\left(\mathcal{I} \mathcal{L}_{g, 1}\right) \rightarrow \wedge^{3} L^{*} \oplus L^{*}
$$

by using the Johnson homomorphism $\tau$ for $\mathcal{I}_{g, 1}$. We can check that $\mathcal{J}$ coincides with the projection to the first two components of the isomorphism in Theorem 3.3. In [8, Section 5.1], Broaddus, Farb and Putman gave another construction of $\mathcal{J}$. In fact, their homomorphisms called relative Johnson homomorphisms cover not only $\mathcal{I} \mathcal{L}_{g, 1}$ but any subgroup of $\mathcal{M}_{g, 1}$ fixing a given submodule of $H$.

\section{The first and second homology of $\operatorname{urSp}(2 g)$}

In this section, we determine the first and second homology of $\operatorname{urSp}(2 g)$ for later use. By a technical reason, we first consider its index 2 subgroup $\operatorname{urSp}^{+}(2 g)$ defined by

$$
1 \longrightarrow \operatorname{urSp}^{+}(2 g) \longrightarrow \operatorname{urSp}(2 g) \stackrel{\text { detoul }}{\longrightarrow} \mathbb{Z}_{2} \longrightarrow 1 \text {. }
$$


By restricting the sequence (4) to $\operatorname{urSp}^{+}(2 g)$, we have a split exact sequence

$$
1 \longrightarrow S^{2} L \longrightarrow \operatorname{urSp}^{+}(2 g) \stackrel{\mathrm{ul}}{\longrightarrow} \mathrm{SL}(g, \mathbb{Z}) \longrightarrow 1 .
$$

Proposition 4.1

for $g \geq 3$.

(2) $H_{2}\left(\operatorname{urSp}^{+}(2 g)\right) \cong \begin{cases}\mathbb{Z}_{2} \oplus \mathbb{Z}_{2} \oplus \mathbb{Z}_{2} \oplus \mathbb{Z}_{2} & g=3, \\ \mathbb{Z}_{2} \oplus \mathbb{Z}_{2} & g=4, \\ \mathbb{Z}_{2} & g \geq 5\end{cases}$

We will prove this proposition by using the Lyndon-Hochschild-Serre spectral sequence

$$
E_{p, q}^{2}=H_{p}\left(\mathrm{SL}(g, \mathbb{Z}) ; H_{q}\left(S^{2} L\right)\right) \Longrightarrow H_{n}\left(\operatorname{urSp}^{+}(2 g)\right)
$$

associated with (9). Before that, we recall the first and second homology of $\operatorname{SL}(g, \mathbb{Z})$. Refer to books of Milnor [26, Sections 5 and 10] and Rosenberg [31, Sections 4.1 and 4.2] for the facts below and generalities of the second homology of groups. The group $\operatorname{SL}(g, \mathbb{Z})$ has a presentation given by

- generators: $\left\{e_{i j} \mid 1 \leq i \leq g, 1 \leq j \leq g\right.$ and $\left.i \neq j\right\}$,

- relations: $\left[e_{i j}, e_{k l}\right]=1 \quad$ if $j \neq k$ and $i \neq l$,

$$
\begin{aligned}
& {\left[e_{i k}, e_{k j}\right]=e_{i j} \quad \text { if } i \neq j \neq k \neq i,} \\
& \left(e_{12} e_{21}^{-1} e_{12}\right)^{4}=1,
\end{aligned}
$$

where $e_{i j}$ corresponds to the matrix whose diagonal entries and $(i, j)$-entry are 1 with the others 0 . From this presentation, we immediately see that $\operatorname{SL}(g, \mathbb{Z})$ is perfect for every $g \geq 3$. The second homology, which is also called the Schur multiplier, of $\operatorname{SL}(g, \mathbb{Z})$ is also known:

$$
H_{2}(\operatorname{SL}(g, \mathbb{Z})) \cong \begin{cases}\mathbb{Z}_{2} \oplus \mathbb{Z}_{2} & g=3,4,(\text { by van der Kallen [35]), } \\ \mathbb{Z}_{2} & g \geq 5,\end{cases}
$$

where van der Kallen also showed in [35] that one summand of $H_{2}(\operatorname{SL}(3, \mathbb{Z})) \cong$ $\mathbb{Z}_{2} \oplus \mathbb{Z}_{2}$ survives in the stable homology $\lim _{g \rightarrow \infty} H_{2}(\operatorname{SL}(g, \mathbb{Z})) \cong K_{2}(\mathbb{Z}) \cong \mathbb{Z}_{2}$ under stabilization, while the other one vanishes in $\mathrm{H}_{2}(\mathrm{SL}(4, \mathbb{Z}))$.

For computations of the zeroth and first homology of a group $G$, we can use any connected CW-complex $X$ with $\pi_{1} X=G$. Let $X_{g}$ be a connected CW-complex associated with the above presentation of $\operatorname{SL}(g, \mathbb{Z})$. Namely $X_{g}$ consists of one vertex, edges $\left\{\left\langle e_{i j}\right\rangle \mid 1 \leq i \leq g, 1 \leq j \leq g\right.$ and $\left.i \neq j\right\}$ and faces

$\left\{\left\langle\left[e_{i j}, e_{k l}\right]\right\rangle \mid j \neq k\right.$ and $\left.i \neq l\right\} \cup\left\{\left\langle\left[e_{i k}, e_{k j}\right] e_{i j}^{-1}\right\rangle \mid\right.$ if $\left.i \neq j \neq k \neq i\right\} \cup\left\{\left\langle\left(e_{12} e_{21}^{-1} e_{12}\right)^{4}\right\rangle\right\}$ 
attached to the 1 -skeleton of $X_{g}$ along the words. We consider $S^{2} L$ to be a local coefficient system on $X_{g}$. The boundary maps

$$
\begin{aligned}
& \partial_{1}: C_{1}\left(X_{g} ; S^{2} L\right) \rightarrow C_{0}\left(X_{g} ; S^{2} L\right) \cong S^{2} L, \\
& \partial_{2}: C_{2}\left(X_{g} ; S^{2} L\right) \rightarrow C_{1}\left(X_{g} ; S^{2} L\right)
\end{aligned}
$$

of the complex $C_{*}\left(X_{g} ; S^{2} L\right)=C_{*}\left(X_{g}\right) \otimes S^{2} L$ are given by

$$
\begin{aligned}
\partial_{1}\left(\left\langle e_{i j}\right\rangle \otimes c\right) & =\left(e_{i j}^{-1}-1\right) c, \\
\partial_{2}\left(\left\langle e_{1} e_{2} \cdots e_{n}\right\rangle \otimes c\right) & =\left\langle e_{1}\right\rangle \otimes c+\left\langle e_{2}\right\rangle \otimes e_{1}^{-1} c+\left\langle e_{3}\right\rangle \otimes\left(e_{1} e_{2}\right)^{-1} c \\
& +\cdots+\left\langle e_{n}\right\rangle \otimes\left(e_{1} e_{2} \cdots e_{n-1}\right)^{-1} c
\end{aligned}
$$

for $c \in S^{2} L$, where $e_{1}, e_{2}, \ldots, e_{n} \in\left\{e_{i j}\right\}_{i, j} \cup\left\{e_{i j}^{-1}\right\}_{i, j}$ and $\left\langle e_{i j}^{-1}\right\rangle \otimes c:=-\left\langle e_{i j}\right\rangle \otimes e_{i j} c$. The action of $\operatorname{SL}(g, \mathbb{Z})$ on $L$ is given by

$$
e_{i j}: x_{k} \mapsto \delta_{j k} x_{i}+x_{k}, \quad e_{i j}^{-1}: x_{k} \mapsto-\delta_{j k} x_{i}+x_{k}
$$

Lemma $4.2 \quad$ (1) $H_{0}\left(\operatorname{SL}(g, \mathbb{Z}) ; S^{2} L\right) \cong\left(S^{2} L\right)_{\operatorname{SL}(g, \mathbb{Z})}=0$ for $g \geq 3$.

(2) $H_{1}\left(\operatorname{SL}(g, \mathbb{Z}) ; S^{2} L\right)=0$ for $g \geq 4$.

Proof Here and hereafter, we suppose that the indices $i, j, k, l$ are distinct from each other. We have

$$
\begin{gathered}
\partial_{1}\left(\left\langle e_{i j}\right\rangle \otimes X_{j}^{2}\right)=\left(e_{i j}^{-1}-1\right) X_{j}^{2}=\left(-x_{i}+x_{j}\right)^{\otimes 2}-X_{j}^{2}=X_{i}^{2}-X_{i j}, \\
\partial_{1}\left(\left\langle e_{i j}\right\rangle \otimes X_{j k}\right)=\left(e_{i j}^{-1}-1\right) X_{j k}=\left(-X_{i k}+X_{j k}\right)-X_{j k}=-X_{i k} .
\end{gathered}
$$

By running $i, j, k$ in $\{1,2, \ldots, g\}$ with $g \geq 3$, we immediately see that $\partial_{1}$ is surjective and (1) holds. To show (2), it suffices to check that $\partial_{1}: C_{1}\left(\operatorname{SL}(g, \mathbb{Z}) ; S^{2} L\right) / \operatorname{Im} \partial_{2} \rightarrow$ $S^{2} L$ is an isomorphism. Assume that $g \geq 4$. $C_{1}\left(\operatorname{SL}(g, \mathbb{Z}) ; S^{2} L\right)$ is generated by elements of types

$\mathrm{I}:\left\langle e_{i j}\right\rangle \otimes X_{i}^{2}, \quad \mathrm{II}:\left\langle e_{i j}\right\rangle \otimes X_{j}^{2}, \quad \mathrm{III}:\left\langle e_{i j}\right\rangle \otimes X_{k}^{2}$,

$\mathrm{IV}:\left\langle e_{i j}\right\rangle \otimes X_{i j}, \quad \mathrm{~V}:\left\langle e_{i j}\right\rangle \otimes X_{j k}, \quad \mathrm{VI}:\left\langle e_{i j}\right\rangle \otimes X_{i l}, \quad \mathrm{VII}:\left\langle e_{i j}\right\rangle \otimes X_{k l}$.

For $c \in S^{2} L$, we have

$\partial_{2}\left(\left\langle\left[e_{i k}, e_{k j}\right] e_{i j}^{-1}\right\rangle \otimes c\right)=\left\langle e_{i k}\right\rangle \otimes\left(1-e_{k j}^{-1} e_{i j}^{-1}\right) c+\left\langle e_{k j}\right\rangle \otimes\left(e_{i k}^{-1}-e_{i j}^{-1}\right) c-\left\langle e_{i j}\right\rangle \otimes c$.

By putting $c=X_{i}^{2}, X_{j}^{2}, X_{k}^{2}, X_{l}^{2}, X_{j k}$ and $X_{j l}$, we see that

(i) $-\left\langle e_{i j}\right\rangle \otimes X_{i}^{2} \quad$ (type I),

(ii) $\left\langle e_{i k}\right\rangle \otimes\left(X_{i j}+X_{j k}-X_{i k}-X_{i}^{2}-X_{k}^{2}\right)+\left\langle e_{k j}\right\rangle \otimes\left(-X_{i}^{2}+X_{i j}\right)-\left\langle e_{i j}\right\rangle \otimes X_{j}^{2}$, 
(iii) $\left\langle e_{k j}\right\rangle \otimes\left(X_{i}^{2}-X_{i k}+X_{k}^{2}\right)-\left\langle e_{i j}\right\rangle \otimes X_{k}^{2}$,

(iv) $-\left\langle e_{i j}\right\rangle \otimes X_{l}^{2} \quad$ (type III),

(v) $\left\langle e_{i k}\right\rangle \otimes\left(X_{i k}+2 X_{k}^{2}\right)+\left\langle e_{k j}\right\rangle \otimes\left(X_{i k}-X_{i j}\right)-\left\langle e_{i j}\right\rangle \otimes X_{j k}$,

(vi) $\left\langle e_{i k}\right\rangle \otimes\left(X_{i l}+X_{k l}\right)+\left\langle e_{k j}\right\rangle \otimes X_{i l}-\left\langle e_{i j}\right\rangle \otimes X_{j l}$

are in $\operatorname{Im} \partial_{2}$. From (i), (iii) and (iv), $-\left\langle e_{k j}\right\rangle \otimes X_{i k}$ (type VI) is in $\operatorname{Im} \partial_{2}$. Also

(vii) $\partial_{2}\left(\left\langle\left[e_{i j}, e_{k l}\right]\right\rangle \otimes X_{l}^{2}\right)=\left\langle e_{i j}\right\rangle \otimes\left(1-e_{k l}^{-1}\right) X_{l}^{2}+\left\langle e_{k l}\right\rangle \otimes\left(e_{i j}^{-1}-1\right) X_{l}^{2}$,

$$
=\left\langle e_{i j}\right\rangle \otimes\left(X_{k l}-X_{k}^{2}\right)
$$

(viii) $\partial_{2}\left(\left\langle\left[e_{i j}, e_{k j}\right]\right\rangle \otimes X_{j}^{2}\right)=\left\langle e_{i j}\right\rangle \otimes\left(-X_{k}^{2}+X_{k j}\right)+\left\langle e_{k j}\right\rangle \otimes\left(X_{i}^{2}-X_{i j}\right)$

are in $\operatorname{Im} \partial_{2}$. From (iv) and (vii), $\left\langle e_{i j}\right\rangle \otimes X_{k l}$ (type VII) is in $\operatorname{Im} \partial_{2}$. Then we can derive from (vi) that

(ix) $\left\langle e_{i k}\right\rangle \otimes X_{k l}-\left\langle e_{i j}\right\rangle \otimes X_{j l} \in \operatorname{Im} \partial_{2}$.

We see from (iv) and (viii) that

(x) $\left\langle e_{i j}\right\rangle \otimes X_{j k}-\left\langle e_{k j}\right\rangle \otimes X_{j i} \in \operatorname{Im} \partial_{2}$.

Finally, we can derive from (ii) and (v) that

(xi) $\left\langle e_{i k}\right\rangle \otimes\left(X_{j k}-X_{i k}-X_{k}^{2}\right)+\left\langle e_{k j}\right\rangle \otimes X_{i j}-\left\langle e_{i j}\right\rangle \otimes X_{j}^{2}$,

(xii) $\left\langle e_{i k}\right\rangle \otimes\left(X_{i k}+2 X_{k}^{2}\right)-\left\langle e_{k j}\right\rangle \otimes X_{i j}-\left\langle e_{i j}\right\rangle \otimes X_{j k}$

are in $\operatorname{Im} \partial_{2}$.

We have so far shown that $C_{1}\left(\operatorname{SL}(g, \mathbb{Z}) ; S^{2} L\right) / \operatorname{Im} \partial_{2}$ is a quotient of the module $M$ generated by the elements of types (II), (IV) and (V) with the relations (ix), (x), (xi) and (xii). We can use (xii) to remove $\left\langle e_{i k}\right\rangle \otimes X_{i k}$ (type IV) and to produce a relation

(xiii) $\left\langle e_{i k}\right\rangle \otimes\left(X_{j k}+X_{k}^{2}\right)-\left\langle e_{i j}\right\rangle \otimes\left(X_{j}^{2}+X_{j k}\right)$

in $M$ from (xi). Therefore $M$ is generated by the elements of types (II) and (V) with the relations (ix), (x), (xiii). The relation (ix) enables us to put $Y_{i l}:=-\left\langle e_{i j}\right\rangle \otimes X_{j l} \in M$, which does not depend on $j$, and the relation (x) shows that $Y_{i l}=Y_{l i}$. On the other hand, if we put $Y_{i}(j, k):=\left\langle e_{i j}\right\rangle \otimes X_{j}^{2}-\left\langle e_{i k}\right\rangle \otimes X_{k j}$, it follows from (ix) and (xiii) 
that $Y_{i}(j, l)=Y_{i}(j, k)=Y_{i}(k, j) \in M$. This implies that $Y_{i}:=Y_{i}(j, l) \in M$ is independent of $j$ and $l$. Consequently, $M$ is a free module with a basis $\left\{Y_{i} \mid 1 \leq i \leq g\right\} \cup$ $\left\{Y_{j k} \mid 1 \leq j<k \leq g\right\}$. It is easy to see that the homomorphism

$$
\tilde{\partial}_{1}: M \rightarrow C_{0}\left(\mathrm{SL}(g, \mathbb{Z}) ; S^{2} L\right) \cong S^{2} L
$$

induced from the surjection

$$
\partial_{1}: C_{1}\left(\operatorname{SL}(g, \mathbb{Z}) ; S^{2} L\right) / \operatorname{Im} \partial_{2} \rightarrow C_{0}\left(\operatorname{SL}(g, \mathbb{Z}) ; S^{2} L\right)
$$

is an isomorphism since $\tilde{\partial}_{1}\left(Y_{i}\right)=X_{i}^{2}$ and $\tilde{\partial}_{1}\left(Y_{j k}\right)=X_{j k}$. Therefore

$$
\partial_{1}: C_{1}\left(\operatorname{SL}(g, \mathbb{Z}) ; S^{2} L\right) / \operatorname{Im} \partial_{2} \rightarrow S^{2} L
$$

is an isomorphism and (2) is proved.

Lemma 4.3 $H_{0}\left(\operatorname{SL}(g, \mathbb{Z}) ; H_{2}\left(S^{2} L\right)\right) \cong\left(\wedge^{2}\left(S^{2} L\right)\right)_{\operatorname{SL}(g, \mathbb{Z})}=0$ for $g \geq 4$.

Proof By definition, the coinvariant part $\left(\wedge^{2}\left(S^{2} L\right)\right)_{S L(g, \mathbb{Z})}$ is the quotient of $\wedge^{2}\left(S^{2} L\right)$ by the submodule $Q_{1}$ generated by $\left\{[e ; x] \mid e \in \operatorname{SL}(g, \mathbb{Z}), x \in \wedge^{2}\left(S^{2} L\right)\right\}$, where we put $[e ; x]:=e x-x$. Direct computations show that

(i) $\left[e_{i j} ; X_{i}^{2} \wedge X_{j}^{2}\right]=X_{i}^{2} \wedge X_{i j}$,

(ii) $\left[e_{k l} ; X_{i}^{2} \wedge X_{j l}\right]=X_{i}^{2} \wedge X_{j k}$,

(iii) $\left[e_{k j} ; X_{i}^{2} \wedge X_{j}^{2}\right]=X_{i}^{2} \wedge\left(X_{k}^{2}+X_{j k}\right)$,

(iv) $\left[e_{j i} ; X_{i}^{2} \wedge X_{j k}\right]=\left(X_{j}^{2}+X_{i j}\right) \wedge X_{j k}$,

(v) $\left[e_{i j} ; X_{j}^{2} \wedge X_{k l}\right]=X_{i}^{2} \wedge X_{k l}+X_{i j} \wedge X_{k l}$

are in $Q_{1}$ and that they generate $\wedge^{2}\left(S^{2} L\right)$ by running $i, j, k, l$ in $\{1,2, \ldots, g\}$ with $g \geq 4$. This completes the proof.

Proof of Proposition 4.1(1) for $g \geq 3$ and (2) for $g \geq 4$ When $g \geq 3$, we have $E_{1,0}^{2}=E_{0,1}^{2}=0$ in the spectral sequence (10) by Lemma $4.2(1)$ and the fact that $H_{1}(\operatorname{SL}(g, \mathbb{Z}))=0$. This proves $(1)$.

Assume further that $g \geq 4$. By Lemma 4.2(2) and Lemma 4.3, we have $E_{1,1}^{2}=E_{0,2}^{2}=0$ in the spectral sequence (10). It follows that $H_{2}\left(\operatorname{urSp}^{+}(2 g)\right) \cong H_{2}(\operatorname{SL}(g, \mathbb{Z}))$. We finish the proof of (2) for $g \geq 4$ by using the explicit description of $H_{2}(\operatorname{SL}(g, \mathbb{Z}))$.

Corollary 4.4 (1) $H_{1}(\operatorname{urSp}(2 g)) \cong H_{1}(\mathrm{GL}(g, \mathbb{Z})) \cong \mathbb{Z}_{2}$ for $g \geq 3$.

(2) $H_{2}(\operatorname{urSp}(2 g)) \cong H_{2}\left(\operatorname{urSp}^{+}(2 g)\right)$ for $g \geq 3$. 
Proof By using the Lyndon-Hochschild-Serre spectral sequence associated with the split extension (8) and the fact that $H_{1}\left(\operatorname{urSp}^{+}(2 g)\right)=0$, we have $H_{1}(\operatorname{urSp}(2 g)) \cong \mathbb{Z}_{2}$ and $H_{2}(\operatorname{urSp}(2 g)) \cong H_{2}\left(\operatorname{urSp}^{+}(2 g)\right)_{\mathbb{Z}_{2}}$. For $g \geq 4$, we have that $H_{2}\left(\operatorname{urSp}^{+}(2 g)\right)_{\mathbb{Z}_{2}}$ $\cong H_{2}(\mathrm{SL}(g, \mathbb{Z}))_{\mathbb{Z}_{2}}$. The action of $\mathbb{Z}_{2}$ on $H_{2}\left(\operatorname{urSp}^{+}(2 g)\right)$ is compatible with that on $H_{2}(\operatorname{SL}(g, \mathbb{Z}))$ and the latter one is known to be trivial. Hence $H_{2}(\operatorname{urSp}(2 g)) \cong$ $H_{2}\left(\operatorname{urSp}^{+}(2 g)\right)$ follows. When $g=3$, the action of $\mathbb{Z}_{2}$ on $H_{2}\left(\operatorname{urSp}^{+}(2 g)\right)$ is also trivial, since we can take the minus of the identity matrix as a lift of the generator of $\mathbb{Z}_{2}$ and it is central. Therefore $H_{2}(\operatorname{urSp}(2 g)) \cong H_{2}\left(\operatorname{urSp}^{+}(2 g)\right)$ holds also for $g=3$.

It remains to compute $\mathrm{H}_{2}\left(\mathrm{urSp}^{+}(2 g)\right)$ for $g=3$.

Lemma 4.5 $H_{1}\left(\operatorname{SL}(3, \mathbb{Z}) ; S^{2} L\right) \cong \mathbb{Z}_{2}$ and it is generated by $\left\langle e_{12}\right\rangle \otimes X_{3}^{2}$.

Sketch of Proof Now $\operatorname{SL}(3, \mathbb{Z})$ has a presentation consisting of 6 generators and 13 relations. Also we have $S^{2} L \cong \mathbb{Z}^{6}$. Hence the complex

$$
C_{2}\left(\mathrm{SL}(3, \mathbb{Z}) ; S^{2} L\right) \stackrel{\partial_{2}}{\rightarrow} C_{1}\left(\mathrm{SL}(3, \mathbb{Z}) ; S^{2} L\right) \stackrel{\partial_{1}}{\rightarrow} C_{0}\left(\mathrm{SL}(3, \mathbb{Z}) ; S^{2} L\right)
$$

can be explicitly written as

$$
\mathbb{Z}^{78 \stackrel{D_{2}}{\longrightarrow}} \mathbb{Z}^{36} \stackrel{D_{1} \cdot}{\longrightarrow} \mathbb{Z}^{6}
$$

with some matrices $D_{1}$ and $D_{2}$. The author with an aid of a computer calculated the homology by using the Smith normal form. We omit the details.

Lemma 4.6 $H_{0}\left(\mathrm{SL}(3, \mathbb{Z}) ; H_{2}\left(S^{2} L\right)\right) \cong H_{2}\left(S^{2} L\right)_{\mathrm{SL}(3, \mathbb{Z})} \cong\left(\wedge^{2}\left(S^{2} L\right)\right)_{\mathrm{SL}(3, \mathbb{Z})} \cong \mathbb{Z}_{2}$ and it is generated by $X_{3}^{2} \wedge X_{2}^{2}$. Moreover this generator is mapped nontrivially to $H_{2}(\mathrm{Sp}(6, \mathbb{Z}))$ by the composition $H_{2}\left(S^{2} L\right)_{\mathrm{SL}(3, \mathbb{Z})} \rightarrow H_{2}\left(\operatorname{urSp}^{+}(6)\right) \rightarrow H_{2}(\mathrm{Sp}(6, \mathbb{Z}))$ induced from the inclusions $S^{2} L \hookrightarrow \operatorname{urSp}^{+}(6) \hookrightarrow \operatorname{Sp}(6, \mathbb{Z})$.

In the proof of this lemma, the following theorem by Stein plays a key role.

Theorem 4.7 (Stein [34, Theorem 2.2]) $H_{2}(\operatorname{Sp}(6, \mathbb{Z})) \cong \mathbb{Z} \oplus \mathbb{Z}_{2}$ and the abelian cycle associated with the homomorphism $\varphi: \mathbb{Z}^{2} \rightarrow \operatorname{Sp}(6, \mathbb{Z})$ defined by

$$
\varphi((1,0))=X_{3}^{2}, \quad \varphi((0,1))=X_{2}^{2}
$$

gives the element of order 2, where $X_{3}^{2}$ and $X_{2}^{2}$ are in $S^{2} L \subset \operatorname{urSp}^{+}(6) \subset \operatorname{Sp}(6, \mathbb{Z})$. 
Proof of Lemma 4.6 We use the same notation as in the proof of Lemma 4.3. The computational results (i), (iii) and (iv) are valid also for $g=3$. In particular, the elements $X_{i}^{2} \wedge X_{i j}, X_{i j} \wedge X_{j k}$ and $X_{i}^{2} \wedge X_{k}^{2}+X_{i}^{2} \wedge X_{j k}$ are in $Q_{1}$. We also see that

$$
\begin{aligned}
& {\left[e_{k i} ; X_{i}^{2} \wedge X_{i j}\right]=X_{k}^{2} \wedge X_{k j}+X_{k}^{2} \wedge X_{i j}+X_{i k} \wedge X_{k j}+X_{i k} \wedge X_{i j}+X_{i}^{2} \wedge X_{k j},} \\
& {\left[e_{j i} ; X_{i}^{2} \wedge X_{i j}\right]=2 X_{i}^{2} \wedge X_{j}^{2}+X_{i j} \wedge X_{j}^{2} .}
\end{aligned}
$$

are in $Q_{1}$, from which $X_{k}^{2} \wedge X_{i j}+X_{i}^{2} \wedge X_{k j}$ and $2 X_{i}^{2} \wedge X_{j}^{2}$ are in $Q_{1}$. Then there remains only two possibilities: $\left(\wedge^{2}\left(S^{2} L\right)\right)_{\mathrm{SL}(3, \mathbb{Z})}=0$ or $\mathbb{Z}_{2}$ generated by

$$
X_{1}^{2} \wedge X_{23}=X_{3}^{2} \wedge X_{12}=X_{2}^{2} \wedge X_{13}=X_{1}^{2} \wedge X_{2}^{2}=X_{1}^{2} \wedge X_{3}^{2}=X_{2}^{2} \wedge X_{3}^{2} .
$$

By using Theorem 4.7, we see that the latter is true. Indeed, the element $X_{2}^{2} \wedge X_{3}^{2}$ just maps to the element of order 2 in $H_{2}(\operatorname{Sp}(6, \mathbb{Z}))$ by the map $\left(\wedge^{2}\left(S^{2} L\right)\right)_{\mathrm{SL}(3, \mathbb{Z})}=$ $H_{2}\left(S^{2} L\right)_{\mathrm{SL}(3, \mathbb{Z})} \rightarrow H_{2}(\mathrm{Sp}(6, \mathbb{Z}))$.

Proof of Proposition 4.1 for $g=3$ The $E^{2}$-term of the Lyndon-Hochschild-Serre spectral sequence associated with the split extension (8) is given as follows:

\begin{tabular}{|c|c|c|c} 
& & & \\
\hline$\left(\wedge^{2}\left(S^{2} L\right)\right)_{\mathrm{SL}(3, \mathbb{Z})} \cong \mathbb{Z}_{2}$ & & & \\
\hline$\left(S^{2} L\right)_{\mathrm{SL}(3, \mathbb{Z})}=0$ & $H_{1}\left(\mathrm{SL}(3, \mathbb{Z}) ; S^{2} L\right) \cong \mathbb{Z}_{2}$ & $H_{2}\left(\operatorname{SL}(3, \mathbb{Z}) ; S^{2} L\right)$ & \\
\hline $\mathbb{Z}$ & $H_{1}(\operatorname{SL}(3, \mathbb{Z}))=0$ & $H_{2}(\operatorname{SL}(3, \mathbb{Z})) \cong \mathbb{Z}_{2}^{2}$ & $H_{3}(\operatorname{SL}(3, \mathbb{Z}))$ \\
\hline
\end{tabular}

By Lemma 4.6, the generator of $\left(\wedge^{2}\left(S^{2} L\right)\right)_{\mathrm{SL}(3, \mathbb{Z})}=\mathbb{Z}_{2}$ survives in $H_{2}\left(\mathrm{urSp}^{+}(6)\right)$. Therefore $d_{2}: H_{2}\left(\operatorname{SL}(3, \mathbb{Z}) ; S^{2} L\right) \rightarrow\left(\wedge^{2}\left(S^{2} L\right)\right)_{\mathrm{SL}(3, \mathbb{Z})}$ is a trivial map. The existence of the splitting of the extension $(8)$ shows that $d_{2}: H_{3}(\operatorname{SL}(3, \mathbb{Z})) \rightarrow H_{1}\left(\operatorname{SL}(3, \mathbb{Z}) ; S^{2} L\right)$ and $d_{3}: H_{3}(\operatorname{SL}(3, \mathbb{Z})) \rightarrow\left(\wedge^{2}\left(S^{2} L\right)\right)_{\mathrm{SL}(3, \mathbb{Z})}$ are also trivial. Hence $E_{p, q}^{2}=E_{p, q}^{\infty}$ for $p+q \leq 2$. The $E^{\infty}$-term says that there exists a filtration

$$
H_{2}\left(\operatorname{urSp}^{+}(6)\right) \supset F_{0} \supset F_{1}=E_{0,2}^{\infty}
$$

with $H_{2}\left(\operatorname{urSp}^{+}(6)\right) / F_{0} \cong E_{2,0}^{\infty}$ and $F_{0} / F_{1} \cong E_{1,1}^{\infty}$. Again the existence of the splitting of the extension (8) shows that $H_{2}\left(\operatorname{urSp}^{+}(6)\right) \cong F_{0} \oplus E_{2,0}^{\infty} \cong F_{0} \oplus H_{2}(\operatorname{SL}(3, \mathbb{Z}))$. Finally we consider the extension

$$
0 \longrightarrow\left(\wedge^{2}\left(S^{2} L\right)\right)_{\mathrm{SL}(3, \mathbb{Z})} \cong \mathbb{Z}_{2} \longrightarrow F_{0} \longrightarrow H_{1}\left(\mathrm{SL}(3, \mathbb{Z}) ; S^{2} L\right) \cong \mathbb{Z}_{2} \longrightarrow 0 .
$$

Suppose $F_{0} \cong \mathbb{Z}_{4}$. Then the second map $\mathbb{Z}_{2} \rightarrow \mathbb{Z}_{4}$ should send $1 \in \mathbb{Z}_{2}$ to $2 \in \mathbb{Z}_{4}$. This contradicts to the fact that the generator of $\left(\wedge^{2}\left(S^{2} L\right)\right)_{\mathrm{SL}(3, \mathbb{Z})}$ maps to the element of order 2 in $H_{2}(\operatorname{Sp}(6, \mathbb{Z})) \cong \mathbb{Z} \oplus \mathbb{Z}_{2}$. Therefore $F_{0} \cong \mathbb{Z}_{2} \oplus \mathbb{Z}_{2}$, finishing the proof. 
Remark 4.8 The homology of $\operatorname{SL}(3, \mathbb{Z})$ was completely determined by Soulé [33]. In particular, $H_{3}(\operatorname{SL}(3, \mathbb{Z})) \cong \mathbb{Z}_{3}^{2} \oplus \mathbb{Z}_{4}^{2}$.

We finish this section by pointing out a byproduct of our argument (see also Remark 5.2). Consider the second homology of the full mapping class group $\mathcal{M}_{3,1}$ of genus 3 . Korkmaz and Stipsicz [22] showed that $H_{2}\left(\mathcal{M}_{3}\right)$ is $\mathbb{Z}$ or $\mathbb{Z} \oplus \mathbb{Z}_{2}$. Now we can use Lemma 3.1 and the fact that the generator of $\left(\wedge^{2}\left(S^{2} L\right)\right)_{\mathrm{SL}(3, \mathbb{Z})} \cong \mathbb{Z}_{2}$ maps to the element of order 2 in $H_{2}(\operatorname{Sp}(6, \mathbb{Z})) \cong \mathbb{Z} \oplus \mathbb{Z}_{2}$ to show that there exists an element of $H_{2}\left(\mathcal{M}_{3,1}\right)$ which comes from $H_{2}\left(\mathcal{I} \mathcal{L}_{g, 1}\right)$ and maps to the element of order 2 in $H_{2}(\operatorname{Sp}(6, \mathbb{Z}))$. Consequently, we have:

Theorem 4.9 $H_{2}\left(\mathcal{M}_{3,1}\right) \cong \mathbb{Z} \oplus \mathbb{Z}_{2}$.

By using an argument of Korkmaz and Stipsicz in [22], we can derive the following.

Corollary 4.10 $H_{2}\left(\mathcal{M}_{3}\right) \cong \mathbb{Z} \oplus \mathbb{Z}_{2}$ and $H_{2}\left(\mathcal{M}_{3, *}\right) \cong \mathbb{Z} \oplus \mathbb{Z} \oplus \mathbb{Z}_{2}$, where $\mathcal{M}_{g, *}$ denotes the mapping class group of a surface of genus $g$ with one puncture.

\section{The first and second homology of $\mathcal{L}_{g, 1}$}

We use our results in the previous sections to determine $H_{1}\left(\mathcal{L}_{g, 1}\right)$ and give a lower bound of $H_{2}\left(\mathcal{L}_{g, 1}\right)$.

\section{Theorem 5.1}

(1) $H_{1}\left(\mathcal{L}_{g, 1}\right) \cong \begin{cases}\mathbb{Z}_{2} \oplus \mathbb{Z}_{2} & g=3, \\ \mathbb{Z}_{2} & g \geq 4 .\end{cases}$

(2) The map $\left(\left.\sigma\right|_{\mathcal{L}_{g, 1}}\right)_{*}: H_{2}\left(\mathcal{L}_{g, 1}\right) \rightarrow H_{2}(\operatorname{urSp}(2 g))$ is surjective for $g \geq 3$.

Proof Consider the five-term exact sequence

$$
\begin{aligned}
& H_{2}\left(\mathcal{L}_{g, 1}\right) \rightarrow H_{2}(\operatorname{urSp}(2 g)) \\
& \quad \rightarrow H_{1}\left(\mathcal{I}_{g, 1}\right)_{\mathrm{urSp}(2 g)} \rightarrow H_{1}\left(\mathcal{L}_{g, 1}\right) \rightarrow H_{1}(\operatorname{urSp}(2 g)) \rightarrow 0
\end{aligned}
$$

associated with the group extension (2). We have seen that $H_{1}(\operatorname{urSp}(2 g)) \cong \mathbb{Z}_{2}$. We now show that

$$
H_{1}\left(\mathcal{I}_{g, 1}\right)_{\mathrm{urSp}(2 g)} \cong \begin{cases}\mathbb{Z}_{2} & g=3, \\ 0 & g \geq 4,\end{cases}
$$

which proves the theorem for $g \geq 4$. 
Put $H_{1}\left(\mathcal{I}_{g, 1}\right)_{\mathrm{urSp}(2 g)}=H_{1}\left(\mathcal{I}_{g, 1}\right) / Q_{2}$ with $Q_{2}$ generated by

$$
\left\{[\sigma ; x] \mid \sigma \in \operatorname{urSp}(2 g), x \in H_{1}\left(\mathcal{I}_{g, 1}\right)\right\} .
$$

Note that $Q_{2}$ includes $Q_{0}$ in the proof of Lemma 3.2 since $S^{2} L \subset \operatorname{urSp}(2 g)$.

We have

$$
\left[e_{k l}^{-1} \oplus e_{l k} ;\left(y_{i} \wedge y_{j} \wedge y_{k}, \bar{y}_{i} \bar{y}_{j} \bar{y}_{k}\right)\right]=\left(y_{i} \wedge y_{j} \wedge y_{l}, \bar{y}_{i} \bar{y}_{j} \bar{y}_{l}\right)
$$

for $g \geq 4$ and also have

$$
\left[e_{i k}^{-1} \oplus e_{k i} ;\left(y_{i} \wedge x_{j} \wedge y_{j}, \bar{y}_{i} \bar{x}_{j} \bar{y}_{j}\right)\right]=\left(y_{k} \wedge x_{j} \wedge y_{j}, \bar{y}_{k} \bar{x}_{j} \bar{y}_{j}\right)
$$

for $g \geq 3$. So $H_{1}\left(\mathcal{I}_{g, 1}\right) / Q_{2}=0$ holds for $g \geq 4$. In the case where $g=3$, we have

$$
\begin{gathered}
{\left[\left(\begin{array}{lll}
0 & 0 & 1 \\
0 & 1 & 0 \\
1 & 0 & 0
\end{array}\right) \oplus\left(\begin{array}{lll}
0 & 0 & 1 \\
0 & 1 & 0 \\
1 & 0 & 0
\end{array}\right) ;\left(y_{1} \wedge y_{2} \wedge y_{3}, \bar{y}_{1} \bar{y}_{2} \bar{y}_{3}\right)\right]} \\
{\left[e_{i k}^{-1} \oplus e_{k i} ;\left(0, \bar{y}_{i} \bar{y}_{j}\right)\right]=\left(0, \bar{y}_{k} \bar{y}_{j}\right) .}
\end{gathered}
$$

Therefore $H_{1}\left(\mathcal{I}_{3,1}\right) / Q_{2}$ is at most $\mathbb{Z}_{2}$ generated by $\left(y_{1} \wedge y_{2} \wedge y_{3}, \bar{y}_{1} \bar{y}_{2} \bar{y}_{3}\right)$. To see that $H_{1}\left(\mathcal{I}_{3,1}\right) / Q_{2} \cong \mathbb{Z}_{2}$, which proves (1) and (2) for $g=3$ simultaneously, we now show that there exists a splitting $H_{1}\left(\mathcal{L}_{3,1}\right) \rightarrow H_{1}\left(\mathcal{I}_{3,1}\right) / Q_{2}$ by constructing a homomorphism $H_{1}\left(\mathcal{L}_{3,1}\right) \rightarrow \mathbb{Z}_{2}$ whose precomposition by $H_{1}\left(\mathcal{I}_{3,1}\right) \rightarrow H_{1}\left(\mathcal{L}_{3,1}\right)$ is nontrivial. Indeed if such a homomorphism exists, $H_{1}\left(\mathcal{I}_{3,1}\right) / Q_{2} \cong \mathbb{Z}_{2}$ immediately follows and the composition $H_{1}\left(\mathcal{I}_{3,1}\right) / Q_{2} \rightarrow H_{1}\left(\mathcal{L}_{3,1}\right) \rightarrow \mathbb{Z}_{2} \cong H_{1}\left(\mathcal{I}_{3,1}\right) / Q_{2}$ becomes the identity map.

Our construction uses the extended Johnson homomorphism

$$
\rho=(\tilde{k}, \sigma): \mathcal{M}_{3,1} \longrightarrow \frac{1}{2} \wedge^{3} H \rtimes \operatorname{Sp}(6, \mathbb{Z})
$$

first defined by Morita [29]. Note that $\tilde{k}: \mathcal{M}_{3,1} \rightarrow \frac{1}{2} \wedge^{3} H$ is a crossed homomorphism which extends the original Johnson homomorphism $\tau: \mathcal{I}_{3,1} \rightarrow \wedge^{3} H$. Precisely speaking, such an extension $\tilde{k}$ is not unique but unique up to certain coboundaries (see [29, Sections 4, 5] for details). Here we use the formulation by Birman, Brendle and Broaddus in [5, Section 2.2] and denote their crossed homomorphism by $\tilde{k}: \mathcal{M}_{3,1} \rightarrow \frac{1}{2} \wedge^{3} H$ again.

Consider the composition

$$
\psi: \mathcal{L}_{3,1} \stackrel{\left.\tilde{k}\right|_{\mathcal{L}_{g, 1}}}{\longrightarrow} \frac{1}{2} \wedge^{3} H \stackrel{\text { proj }}{\longrightarrow} \frac{1}{2} \wedge^{3} L \cong \frac{1}{2} \wedge^{3} \mathbb{Z}^{3} \cong \frac{1}{2} \mathbb{Z} \longrightarrow\left(\frac{1}{2} \mathbb{Z}\right) /(2 \mathbb{Z}),
$$


where the second map is induced from the projection $H \rightarrow L$ (in other word, this map assigns the coefficient of $y_{1} \wedge y_{2} \wedge y_{3}$ under our basis of $H$ ). We claim the following:

(i) $\operatorname{Im} \psi \subset \mathbb{Z} / 2 \mathbb{Z}=\mathbb{Z}_{2}$,

(ii) $\psi: \mathcal{L}_{3,1} \rightarrow \mathbb{Z}_{2}$ is a homomorphism,

(iii) the composition $\mathcal{I}_{3,1} \rightarrow \mathcal{L}_{3,1} \stackrel{\psi}{\rightarrow} \mathbb{Z}_{2}$ is nontrivial.

To show (i), we recall that $\mathcal{L}_{3,1}=\mathcal{I}_{3,1} \mathcal{H}_{3,1}$, where $\mathcal{H}_{3,1}$ is the preimage of the handlebody mapping class group $\mathcal{H}_{3}$ of genus 3 by the natural homomorphism $\mathcal{M}_{3,1} \rightarrow \mathcal{M}_{3}$. Birman, Brendle and Broaddus showed in [5, Section 2.2] that $\tilde{k}(h)$ does not have the term $n y_{1} \wedge y_{2} \wedge y_{3}$ with $n \in \frac{1}{2} \mathbb{Z}-\{0\}$ for any $h \in \mathcal{H}_{3,1}$. Since

$$
\tilde{k}(f)=\tilde{k}(i h)=\tilde{k}(i)+{ }^{\sigma(i)} \tilde{k}(h)=\tilde{k}(i)+\tilde{k}(h)
$$

for any element $f=i h \in \mathcal{L}_{3,1}$ with $i \in \mathcal{I}_{3,1}$ and $h \in \mathcal{L}_{3,1}$, and $\tilde{k}(i)=\tau(i) \in \wedge^{3} H$, we see that $\psi(f)=\psi(i)+\psi(h)=\psi(i) \in \mathbb{Z} / 2 \mathbb{Z}$, which proves (i). Next, (ii) follows from the facts that $\mathcal{L}_{3,1}$ acts on $H$ with keeping $L$ and acts on $L$ through $\left.\mathrm{ul} \circ \sigma\right|_{\mathcal{L}_{g}}: \mathcal{L}_{3,1} \rightarrow$ $\operatorname{GL}(3, \mathbb{Z})$ and that $\operatorname{GL}(3, \mathbb{Z})$ acts on $\wedge^{3} L \cong \mathbb{Z}$ through det: $\operatorname{GL}(3, \mathbb{Z}) \rightarrow\{1,-1\}$. Finally, (iii) clearly follows from the construction and we finish the proof.

Remark 5.2 The above computation of $H_{1}\left(\mathcal{I}_{3,1}\right)_{\mathrm{urSp}(6)}$ and the equality

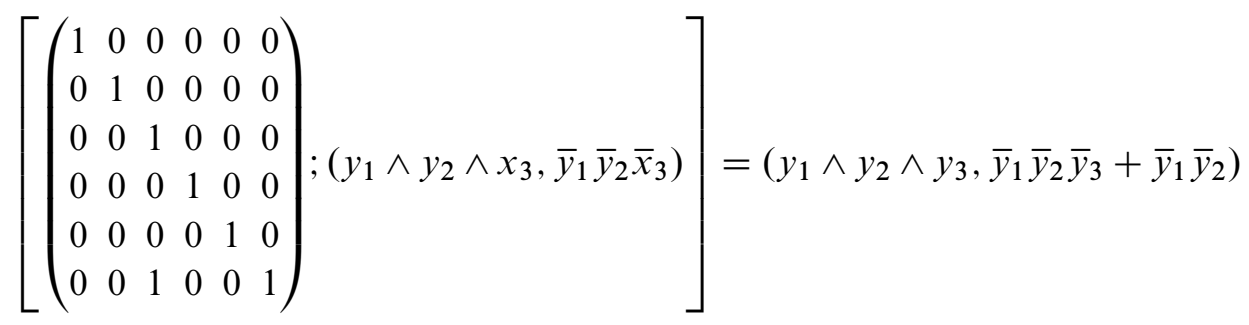

show that $H_{1}\left(\mathcal{I}_{3,1}\right)_{\mathrm{Sp}(6, \mathbb{Z})}=0$ (see also Putman [30, Lemma 6.4]). Then by the five term exact sequence associated with (1), the map $H_{2}\left(\mathcal{M}_{3,1}\right) \rightarrow H_{2}(\operatorname{Sp}(6, \mathbb{Z}))$ is onto. Therefore, by using the results of Korkmaz-Stipsicz and Stein mentioned in Section 4, we can obtain another proof of $H_{2}\left(\mathcal{M}_{3,1}\right) \cong \mathbb{Z} \oplus \mathbb{Z}_{2}$.

Remark 5.3 We saw that $\lim _{g \rightarrow \infty} H_{2}(\operatorname{urSp}(2 g)) \cong \lim _{g \rightarrow \infty} H_{2}(\operatorname{GL}(g, \mathbb{Z}))$ in $\operatorname{Sec}$ tion 4. The stable homology $\lim _{g \rightarrow \infty} H_{2}(\mathrm{GL}(g, \mathbb{Z})) \cong \mathbb{Z}_{2}$ also relates to the second homology of the automorphism group of a free group as shown by Gersten [14]. 


\section{Results for Lagrangian mapping class groups of closed sur- faces}

We now consider the Lagrangian mapping class groups $\mathcal{L}_{g}$ and $\mathcal{I} \mathcal{L}_{g}$ for closed surfaces. The relationship of $\mathcal{L}_{g, 1}$ and $\mathcal{L}_{g}$ is given by the exact sequence

$$
1 \longrightarrow \pi_{1}\left(T_{1} \Sigma_{g}\right) \longrightarrow \mathcal{L}_{g, 1} \longrightarrow \mathcal{L}_{g} \longrightarrow 1,
$$

where $T_{1} \Sigma_{g}$ is the unit tangent bundle of $\Sigma_{g}$ (see Birman [3]), and the relationship of $\mathcal{I} \mathcal{L}_{g, 1}$ and $\mathcal{I} \mathcal{L}_{g}$ is obtained by replacing $\mathcal{L}_{g, 1}$ and $\mathcal{L}_{g}$ with $\mathcal{I} \mathcal{L}_{g, 1}$ and $\mathcal{I} \mathcal{L}_{g}$. As a subgroup of $\mathcal{L}_{g, 1}$ and $\mathcal{I} \mathcal{L}_{g, 1}$, the group $\pi_{1}\left(T_{1} \Sigma_{g}\right) \subset \mathcal{I}_{g, 1}$ is generated by the Dehn twist along the boundary curve of $\Sigma_{g, 1}$ and spin-maps (see Birman [3, Theorem 4.3] and Johnson [19, Section 3], for example).

Theorem 6.1 The homology group $H_{1}\left(\mathcal{I} \mathcal{L}_{g}\right)$ is given by

$$
H_{1}\left(\mathcal{I} \mathcal{L}_{g}\right) \cong \begin{cases}\wedge^{3} L^{*} \oplus \wedge^{2}\left(L^{*} \otimes \mathbb{Z}_{2}\right) \oplus S^{2} L & g=3, \\ \wedge^{3} L^{*} \oplus S^{2} L & g \geq 4\end{cases}
$$

Proof We have an exact sequence

$$
H_{1}\left(\pi_{1}\left(T_{1} \Sigma_{g}\right)\right) \longrightarrow H_{1}\left(\mathcal{I} \mathcal{L}_{g, 1}\right) \longrightarrow H_{1}\left(\mathcal{I} \mathcal{L}_{g}\right) \longrightarrow 0 .
$$

In [23, Section 3.4], Levine showed that $\pi_{1}\left(T_{1} \Sigma_{g}\right)$ projects trivially on $\wedge^{3} L^{*}$ and onto on $L^{*}$ with respect to the abelianization

$$
H_{1}\left(\mathcal{I} \mathcal{L}_{g, 1}\right) \cong \begin{cases}\wedge^{3} L^{*} \oplus L^{*} \oplus \wedge^{2}\left(L^{*} \otimes \mathbb{Z}_{2}\right) \oplus S^{2} L & g=3, \\ \wedge^{3} L^{*} \oplus L^{*} \oplus S^{2} L & g \geq 4 .\end{cases}
$$

Since $\pi_{1}\left(T_{1} \Sigma_{g}\right)$ is included in $\mathcal{I}_{g, 1}$, it projects trivially on $S^{2} L$. Hence the theorem for $g \geq 4$ holds. In the case where $g=3$, we can directly check that all of generators of $\pi_{1}\left(T_{1} \Sigma_{3}\right)$ are sent to $0 \in \wedge^{2}\left(L^{*} \otimes \mathbb{Z}_{2}\right)$, which completes the proof for $g=3$.

\section{Theorem 6.2}

(1) $H_{1}\left(\mathcal{L}_{g}\right) \cong H_{1}\left(\mathcal{L}_{g, 1}\right) \cong \begin{cases}\mathbb{Z}_{2} \oplus \mathbb{Z}_{2} & g=3 \\ \mathbb{Z}_{2} & g \geq 4 .\end{cases}$

(2) The map $\left(\left.\sigma\right|_{\mathcal{L}_{g}}\right)_{*}: H_{2}\left(\mathcal{L}_{g}\right) \rightarrow H_{2}(\operatorname{urSp}(2 g))$ is surjective for $g \geq 3$.

Proof Since $\left.\sigma\right|_{\mathcal{L}_{g, 1}}: \mathcal{L}_{g, 1} \rightarrow \operatorname{urSp}(2 g)$ factors through $\mathcal{L}_{g}$, (1) for $g \geq 4$ immediately holds. (1) for $g=3$ also holds by explicit computations of the extended Johnson homomorphism for generators of $\pi_{1}\left(T_{1} \Sigma_{3}\right)$. The proof of (2) is the same as that of Theorem 5.1. 


\section{Remarks on higher (co)homology of $\mathcal{L}_{g}$ and $\mathcal{I} \mathcal{L}_{g}$}

\subsection{Relationship to the homology of the pure braid group}

In [23], Levine studied various embeddings of the pure braid group $P_{n}$ of $n$ strands into $\mathcal{M}_{g, 1}$ and $\mathcal{M}_{g}$, where $n=g, 2 g$ etc. We now use one of them defined as follows. Let $D_{g}$ be a disk with $g$ holes. We take an embedding $\iota: D_{g} \hookrightarrow \Sigma_{g, 1}$ as in Figure 3, where we consider the surface $\Sigma_{g, 1}$ to be a disk with $g$ handles attached and the belt circles of the handles correspond to the loops $x_{1}, x_{2}, \ldots, x_{g}$ in Figure 1 after filling the boundary $\partial \Sigma_{g, 1}$ by a disk. The mapping class group of $D_{g}$, where the self-diffeomorphisms of $D_{g}$ are supposed to fix the boundary pointwise, is known to be isomorphic to the framed pure braid group of $g$ strands. Here the framing counts how many times one gives Dehn twists along each of the loops parallel to the inner boundary. For any choice of framings, we have a homomorphism from the pure braid group $P_{g}$ of $g$ strands to $\mathcal{M}_{g, 1}$ by extending each mapping class by identity on the outside of $\iota\left(D_{g}\right)$. We can easily check that the image of this map is contained in $\mathcal{I} \mathcal{L}_{g, 1}$. Therefore we obtain a homomorphism $\Phi: P_{g} \rightarrow \mathcal{I} \mathcal{L}_{g, 1}$. Similarly, we have a homomorphism from $P_{g}$ to $\mathcal{I} \mathcal{L}_{g}$ also denoted by $\Phi: P_{g} \rightarrow \mathcal{I} \mathcal{L}_{g}$.

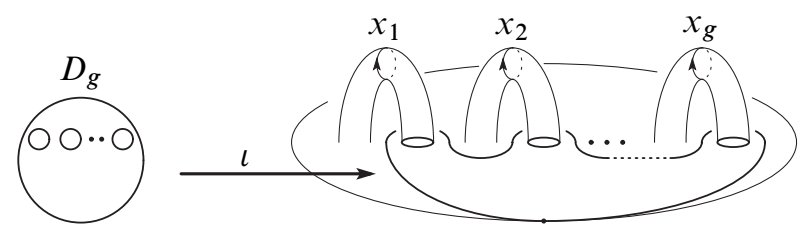

Figure 3. The embedding $\iota: D_{g} \hookrightarrow \Sigma_{g, 1}$

Theorem 7.1 The induced map $\Phi_{*}: H_{*}\left(P_{g}\right) \rightarrow H_{*}\left(\mathcal{I} \mathcal{L}_{g}\right)$ is injective.

Proof Consider the induced map $H^{*}\left(S^{2} L\right) \rightarrow H^{*}\left(P_{g}\right)$ of the composition $P_{g} \stackrel{\Phi}{\rightarrow}$ $\mathcal{I} \mathcal{L}_{g} \rightarrow S^{2} L$ on cohomology. Here the ring structure of $H^{*}\left(P_{g}\right)$ was completely determined by Arnol'd [2], and in particular, it was shown that $H^{*}\left(P_{g}\right)$ is a finitely generated free abelian group and is generated by degree 1 elements as a ring. The former shows that $H_{*}\left(P_{g}\right)$ is also finitely generated free abelian and the latter shows that $H^{*}\left(S^{2} L\right) \rightarrow H^{*}\left(P_{g}\right)$ is onto since it is clear from a presentation of $P_{g}$ (see [3] for example) that $H^{1}\left(S^{2} L\right) \rightarrow H^{1}\left(P_{g}\right)$ is onto. By passing to homology, we see that $H_{*}\left(P_{g}\right) \rightarrow H_{*}\left(S^{2} L\right)$ is injective. The theorem follows from this. 


\subsection{Vanishing of odd Miller-Morita-Mumford classes on $\mathcal{L}_{g}$}

Finally, we discuss the rational cohomology of higher degrees of $\mathcal{L}_{g}$ with relationships to characteristic classes of oriented $\Sigma_{g}$-bundles called Miller-Morita-Mumford classes.

Here we recall the definition of Miller-Morita-Mumford classes following Morita [28]. Let $\pi: E \rightarrow B$ be an oriented $\Sigma_{g}$-bundle over a closed oriented manifold $B$. Since $\Sigma_{g}$ is 2 -dimensional, the relative tangent bundle $\operatorname{Ker} \pi_{*}$ is a vector bundle over $E$ of rank 2. In particular, we can take its Euler class $e \in H^{2}(E)$. Then $i$-th MillerMorita-Mumford class $e_{i}$ is defined by

$$
e_{i}:=\pi_{!}\left(e^{i+1}\right) \in H^{2 i}(B),
$$

where $\pi !: H^{*}(E) \rightarrow H^{*-2}(B)$ is the Gysin map. This construction is natural with respect to bundle maps, so that we can regard $e_{i}$ as a cohomology class in the classifying space. Namely $e_{i} \in H^{2 i}\left(B \operatorname{Diff}_{+} \Sigma_{g}\right)$, where $B \operatorname{Diff}_{+} \Sigma_{g}$ is the classifying space of the topological group Diff $+\Sigma_{g}$ of orientation preserving self-diffeomorphisms of $\Sigma_{g}$ with $C^{\infty}$-topology. By a theorem of Earle and Eells [12], we have $B \operatorname{Diff}_{+} \Sigma_{g}=K\left(\mathcal{M}_{g}, 1\right)$. Therefore

$$
e_{i} \in H^{2 i}\left(B \operatorname{Diff}_{+} \Sigma_{g}\right)=H^{2 i}\left(K\left(\mathcal{M}_{g}, 1\right)\right)=H^{2 i}\left(\mathcal{M}_{g}\right) .
$$

Now we ask whether $e_{i} \in H^{2 i}\left(\mathcal{M}_{g} ; \mathbb{Q}\right)$, regarded as a rational cohomology class, survives in $H^{2 i}\left(\mathcal{I}_{g} ; \mathbb{Q}\right)$ by the pullback of $\mathcal{I}_{g} \hookrightarrow \mathcal{M}_{g}$. A partial answer to this question is given as follows (see Morita [28]). It is known that every odd class $e_{2 i-1} \in$ $H^{4 i-2}\left(\mathcal{M}_{g} ; \mathbb{Q}\right)$ can be obtained as the pullback of some class in $H^{4 i-2}(\operatorname{Sp}(2 g, \mathbb{Z}) ; \mathbb{Q})$, which implies that all the odd classes $e_{2 i-1}$ vanish in $H^{4 i-2}\left(\mathcal{I}_{g} ; \mathbb{Q}\right)$. However, this argument says nothing about even classes $e_{2 i}$ and it has been a long standing problem to determine whether even classes $e_{2 i}$ vanish or not in $H^{4 i}\left(\mathcal{I}_{g} ; \mathbb{Q}\right)$.

The author's motivation for the study in this paper is to attack this problem by considering groups locating between $\mathcal{M}_{g}$ and $\mathcal{I}_{g}$ and investigating the behavior of $e_{i}$ on them. As examples of such a kind of groups, finite index subgroups including level $L$ mapping class groups defined as the kernel of the composition

$$
\mathcal{M}_{g} \longrightarrow \mathrm{Sp}(2 g, \mathbb{Z}) \longrightarrow \mathrm{Sp}(2 g, \mathbb{Z} / L \mathbb{Z})
$$

are often studied. However, we cannot solve the above problem by using them since for any finite index subgroup $G$ of $\mathcal{M}_{g}$ there exists a transfer map

$$
\operatorname{tr}: H^{*}(G ; \mathbb{Q}) \longrightarrow H^{*}\left(\mathcal{M}_{g} ; \mathbb{Q}\right)
$$

such that $\operatorname{tr} \circ i^{*}: H^{*}\left(\mathcal{M}_{g} ; \mathbb{Q}\right) \rightarrow H^{*}\left(\mathcal{M}_{g} ; \mathbb{Q}\right)$ is the multiplication by a positive integer $\left[\mathcal{M}_{g}: G\right]$, where $i: G \hookrightarrow \mathcal{M}_{g}$ denotes the inclusion. In particular, we see that 
the pullback map on the rational cohomology is always injective for any finite index subgroup. Therefore we shall need infinite index subgroups and we focus on $\mathcal{L}_{g}$ and $\mathcal{I} \mathcal{L}_{g}$ in this paper. At present, we cannot give the final answer even for $\mathcal{L}_{g}$, but we now present an observation for odd classes, by which we finish this paper.

Lemma 7.2 If $g$ is sufficiently larger than $q$, we have

$$
H^{q}(\operatorname{urSp}(2 g) ; \mathbb{Q}) \cong H^{q}(\mathrm{GL}(g, \mathbb{Z}) ; \mathbb{Q}) .
$$

Proof The $E_{2}$-term of the Lyndon-Hochschild-Serre spectral sequence for the group extension (4) is given by

$$
E_{2}^{p, q}=H^{p}\left(\mathrm{GL}(g, \mathbb{Z}) ; H^{q}\left(S^{2} L ; \mathbb{Q}\right)\right) .
$$

Our claim immediately follows once we show that $H^{p}\left(\mathrm{GL}(g, \mathbb{Z}) ; H^{q}\left(S^{2} L ; \mathbb{Q}\right)\right)=0$ if $q \geq 1$. Since $H^{q}\left(S^{2} L ; \mathbb{Q}\right) \cong \wedge^{q}\left(S^{2}\left(L^{*} \otimes \mathbb{Q}\right)\right)$ and it is easy to show that the invariant part $\wedge^{q}\left(S^{2}\left(L^{*} \otimes \mathbb{Q}\right)\right)^{\mathrm{GL}(g, \mathbb{Z})}$ is trivial, we can use Borel's vanishing theorem [7] to show that

$$
H^{p}\left(\mathrm{GL}(g, \mathbb{Z}) ; H^{q}\left(S^{2} L ; \mathbb{Q}\right)\right)=0
$$

for any $q \geq 1$.

Theorem 7.3 For every $i$, the $(2 i-1)$-st Miller-Morita-Mumford class $e_{2 i-1}$ vanishes in $H^{4 i-2}\left(\mathcal{L}_{g} ; \mathbb{Q}\right)$ if $g$ is sufficiently larger than $i$.

Proof It is known that the group cohomology $H^{*}(G)$ of a discrete group $G$ can be rewritten as $H^{*}\left(B G^{\delta}\right)$, where $B G$ denotes the classifying space of $G$. When $G$ is a Lie group, we write $G^{C^{\infty}}$ for $G$ with $C^{\infty}$ topology and $G^{\delta}$ for $G$ with discrete topology.

Consider the commutative diagram

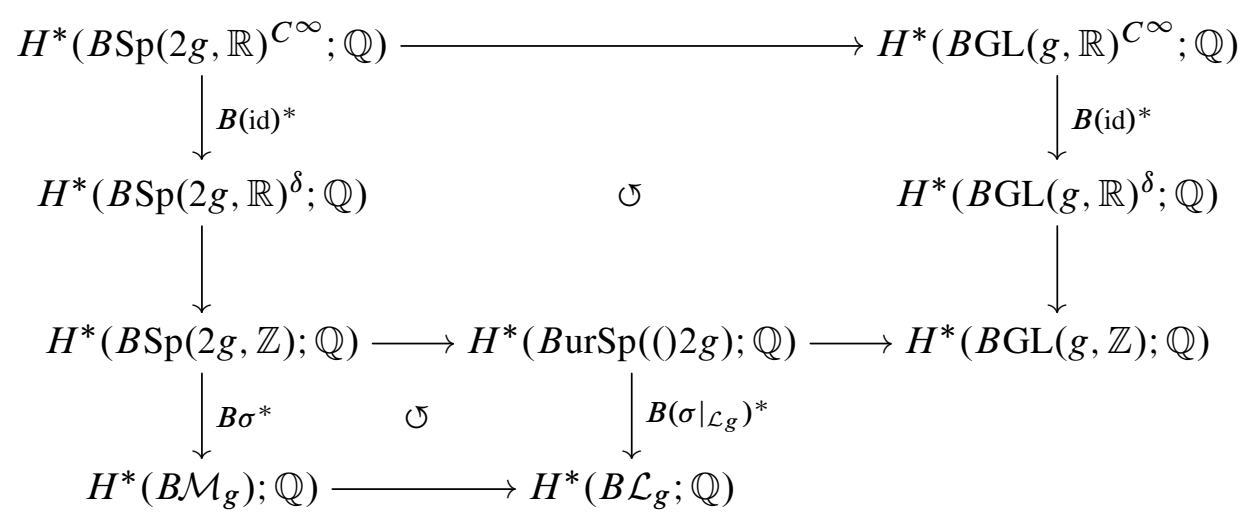


where id denotes the identity map, which always gives a continuous map from $G$ with discrete topology to that with $C^{\infty}$ topology for a Lie group $G$, and all the arrows not labeled are pullbacks by the induced maps of inclusions on classifying spaces. We now assume that $g$ is sufficiently large. Since $\operatorname{Sp}(2 g, \mathbb{R})^{C^{\infty}}$ is homotopy equivalent to the unitary group $U(g)^{C^{\infty}}$, we have $H^{*}\left(B \operatorname{Sp}(2 g, \mathbb{R})^{C^{\infty}} ; \mathbb{Q}\right) \cong H^{*}\left(B U(g)^{C^{\infty}} ; \mathbb{Q}\right)$ and the latter is known to be isomorphic to the polynomial algebra $\mathbb{Q}\left[c_{1}, c_{2}, \ldots\right]$ generated by the Chern classes $c_{1}, c_{2}, \ldots$ independently in the stable range. This polynomial algebra $\mathbb{Q}\left[c_{1}, c_{2}, \ldots\right]$ is mapped onto $\mathbb{Q}\left[c_{1}, c_{3}, c_{5}, \ldots\right]$ in $H^{*}\left(B \operatorname{Sp}(2 g, \mathbb{R})^{\delta} ; \mathbb{Q}\right)$, and onto $\mathbb{Q}\left[e_{1}, e_{3}, e_{5}, \ldots\right]$ in $H^{*}\left(B \mathcal{M}_{g} ; \mathbb{Q}\right)$. We refer to Morita [28] again for these arguments. On the other hand, it was shown by Milnor [27, Appendix] that

$$
B(\mathrm{id})^{*}: H^{*}\left(B \mathrm{GL}(g, \mathbb{R})^{C^{\infty}} ; \mathbb{Q}\right) \longrightarrow H^{*}\left(B \mathrm{GL}(g, \mathbb{R})^{\delta} ; \mathbb{Q}\right)
$$

is trivial for $* \geq 1$. By combining this fact with Lemma 7.2, the theorem follows.

Remark 7.4 Recently, Giansiracusa and Tillmann [15] have proved a closely related result that odd Miller-Morita-Mumford classes vanish in the integral cohomology of the handlebody subgroup $\mathcal{H}_{g}$ for $g \geq 2$. In fact, they showed that odd Miller-MoritaMumford classes are in the kernel of the pullback map on the integral cohomology by $B \operatorname{Diff}_{+} M \rightarrow B \operatorname{Diff}_{+} \Sigma_{g}$ where $M$ is any compact oriented 3-manifold $M$ with $\partial M=\Sigma_{g}$.

Acknowledgements The author would like to express his gratitude to Professor Sadayoshi Kojima and Professor Shigeyuki Morita for their encouragement and to Yusuke Kuno and Andrew Putman for useful discussions and comments. The author also would like to thank the referee for his-or-her helpful comments to improve the paper. This research was supported by JSPS Research Fellowships for Young Scientists and KAKENHI (numbers 08J02356 and 21740044).

\section{References}

[1] J E Andersen, A J Bene, J-B Meilhan, R C Penner, Finite type invariants and fatgraphs, Adv. Math. 225 (2010) 2117-2161 MR2680201

[2] V I Arnol'd, The cohomology ring of the group of dyed braids, Mat. Zametki 5 (1969) 227-231 MR0242196

[3] J S Birman, Braids, links, and mapping class groups, Ann. of Math. Studies 82, Princeton Univ. Press (1974) MR0375281

[4] JS Birman, On the equivalence of Heegaard splittings of closed, orientable 3manifolds, from: "Knots, groups, and 3-manifolds (Papers dedicated to the memory of R. H. Fox)", (L P Neuwirth, editor), Ann. of Math. Studies 84, Princeton Univ. Press (1975) 137-164 MR0375318 
[5] J S Birman, T E Brendle, N Broaddus, Calculating the image of the second JohnsonMorita representation, from: "Groups of diffeomorphisms", (R Penner, D Kotschick, T Tsuboi, N Kawazumi, T Kitano, Y Mitsumatsu, editors), Adv. Stud. Pure Math. 52, Math. Soc. Japan, Tokyo (2008) 119-134 MR2509709

[6] J S Birman, R Craggs, The $\mu$-invariant of 3-manifolds and certain structural properties of the group of homeomorphisms of a closed, oriented 2-manifold, Trans. Amer. Math. Soc. 237 (1978) 283-309 MR0482765

[7] A Borel, Stable real cohomology of arithmetic groups II, from: "Manifolds and Lie groups (Notre Dame, Ind., 1980)", (S Murakami, J Hano, K Okamoto, A Morimoto, H Ozeki, editors), Progr. Math. 14, Birkhäuser, Mass. (1981) 21-55 MR642850

[8] N Broaddus, B Farb, A Putman, Irreducible Sp-representations and subgroup distortion in the mapping class group, Comment. Math. Helv. 86 (2011) 537-556 MR2803852

[9] KS Brown, Cohomology of groups, Graduate Texts in Math. 87, Springer, New York (1982) MR672956

[10] D Cheptea, K Habiro, G Massuyeau, A functorial LMO invariant for Lagrangian cobordisms, Geom. Topol. 12 (2008) 1091-1170 MR2403806

[11] D Cheptea, T T Q Le, A TQFT associated to the LMO invariant of three-dimensional manifolds, Comm. Math. Phys. 272 (2007) 601-634 MR2304469

[12] C J Earle, J Eells, The diffeomorphism group of a compact Riemann surface, Bull. Amer. Math. Soc. 73 (1967) 557-559 MR0212840

[13] S Garoufalidis, J Levine, Finite type 3-manifold invariants, the mapping class group and blinks, J. Differential Geom. 47 (1997) 257-320 MR1601612

[14] S M Gersten, A presentation for the special automorphism group of a free group, J. Pure Appl. Algebra 33 (1984) 269-279 MR761633

[15] J Giansiracusa, U Tillmann, Vanishing of universal characteristic classes for handlebody groups and boundary bundles, J. Homotopy Relat. Struct. 6 (2011) 103-112 MR2818701

[16] S Hirose, The action of the handlebody group on the first homology group of the surface, Kyungpook Math. J. 46 (2006) 399-408 MR2261393

[17] D Johnson, An abelian quotient of the mapping class group $\mathcal{I}_{g}$, Math. Ann. 249 (1980) 225-242 MR579103

[18] D Johnson, Quadratic forms and the Birman-Craggs homomorphisms, Trans. Amer. Math. Soc. 261 (1980) 235-254 MR576873

[19] D Johnson, The structure of the Torelli group I: A finite set of generators for $\mathcal{I}$, Ann. of Math. 118 (1983) 423-442 MR727699

[20] D Johnson, The structure of the Torelli group II: A characterization of the group generated by twists on bounding curves, Topology 24 (1985) 113-126 MR793178 
[21] D Johnson, The structure of the Torelli group III: The abelianization of $\mathcal{I}_{g}$, Topology 24 (1985) 127-144 MR793179

[22] M Korkmaz, A I Stipsicz, The second homology groups of mapping class groups of oriented surfaces, Math. Proc. Cambridge Philos. Soc. 134 (2003) 479-489 MR1981213

[23] J Levine, Pure braids, a new subgroup of the mapping class group and finite-type invariants of 3-manifolds, from: "Tel Aviv Topology Conference: Rothenberg Festschrift (1998)”, (M Farber, W Lück, S Weinberger, editors), Contemp. Math. 231, Amer. Math. Soc. (1999) 137-157 MR1705580

[24] J Levine, Homology cylinders: an enlargement of the mapping class group, Algebr. Geom. Topol. 1 (2001) 243-270 MR1823501

[25] J Levine, The Lagrangian filtration of the mapping class group and finite-type invariants of homology spheres, Math. Proc. Cambridge Philos. Soc. 141 (2006) 303-315 MR2265877

[26] J Milnor, Introduction to algebraic K-theory, Ann. of Math. Studies 72, Princeton Univ. Press (1971) MR0349811

[27] J Milnor, On the homology of Lie groups made discrete, Comment. Math. Helv. 58 (1983) 72-85 MR699007

[28] S Morita, Characteristic classes of surface bundles, Invent. Math. 90 (1987) 551-577 MR914849

[29] S Morita, The extension of Johnson's homomorphism from the Torelli group to the mapping class group, Invent. Math. 111 (1993) 197-224 MR1193604

[30] A Putman, The Picard group of the moduli space of curves with level structures arXiv:0908.0555

[31] J Rosenberg, Algebraic K-theory and its applications, Graduate Texts in Math. 147, Springer, New York (1994) MR1282290

[32] T Sakasai, The Johnson homomorphism and the third rational cohomology group of the Torelli group, Topology Appl. 148 (2005) 83-111 MR2118957

[33] C Soulé, The cohomology of $\mathrm{SL}_{3}(\mathbf{Z})$, Topology 17 (1978) 1-22 MR0470141

[34] M R Stein, The Schur multipliers of $\operatorname{Sp}_{6}(\mathbf{Z}), \operatorname{Spin}_{8}(\mathbf{Z}), \operatorname{Spin}_{7}(\mathbf{Z})$, and $F_{4}(\mathbf{Z})$, Math. Ann. 215 (1975) 165-172 MR0372056

[35] W van der Kallen, The Schur multipliers of $\operatorname{SL}(3, \mathbf{Z})$ and $\operatorname{SL}(4, \mathbf{Z})$, Math. Ann. 212 (1974/75) 47-49 MR0354893

Department of Mathematics, Tokyo Institute of Technology

2-12-1 Oh-okayama, Meguro-ku, 152-8551 Tokyo, Japan

sakasai@math.titech.ac.jp

Received: 20 November $2010 \quad$ Revised: 1 November 2011 